\title{
Some flow visualization experiments on the starting vortex
}

\author{
By D. I. PULLIN AND A. E. PERRY \\ Department of Mechanical Engineering, University of Melbourne, \\ Parkville, Victoria, 3052 Australia
}

(Received 4 December 1978)

\begin{abstract}
A simple dye in water method has been used to visualize the growth of a two-dimensional starting flow vortex formed at a wedge-like sharp edge. Several cases were tested corresponding to different wedge angles and to different values of the time exponent in the velocity-time power law describing the starting flow. Photographic sequences showing the time-wise primary vortex growth are presented from which various secondary-flow details are identified. For the larger wedge angles these include a strong secondary vortex and in some cases a small separation bubble-like flow region immediately adjacent to the wedge apex. For a thin-wedge model the formation of what might be interpreted as small rotation centres along the outer turns of the primaryvortex shear layer is observed but these are not seen as a manifestation of an instability phenomenon in the fluid. Measurements of the trajectories of the primary-vortex centre are compared with the predictions of an inviscid similarity theory of the vortex growth. Although the appropriate Reynolds number in the present experiments was relatively low, comparison between theory and experiments is regarded as reasonable with differences being attributed to viscous effects absent in the similarity theory, and also to apparatus wall effects.
\end{abstract}

\section{Introduction}

The formation of a growing vortex from a salient edge in a flow starting from rest is well known as the basic mechanism for the initial generation of circulation around aerofoils and other bodies. It has been established that the sharp edge fixes the point of boundary-layer separation leading to the familiar coherent spiral shear-layer roll-up characteristic of vortex development. Although essentially transient, such flows are of basic interest as a fundamental means of studying free shear layer and vortex behaviour. Furthermore the vortex formation process is central in understanding the structure of vortex rings (Saffmann 1978; Pullin 1979) and the close analogy between sharp-edge starting flows and leading-edge separation from slender bodies is well known.

The aluminium particle flow visualizations of Prandtl and Tietjens reproduced in Batchelor (1970) are perhaps the first documented photographic evidence of edge vortex formation. Later studies of the phenomenon in an incompressible fluid include those of Wedemeyer (1956) who observed vortex growth behind a moving flat plate and Pierce (1961) who employed spark shadowgraph/heated-vapour methods to view and measure the growth rates of vortices produced by several geometrical configurations. Rott (1956) discussed the similarity laws relating the initial generally nonlinear 
grow th of the vortex in an inviscid fluid to the edge-forming angle and to the velocitytime history of the generating flow. Numerical solutions to the integro-differential equation obtained from these laws and which determine the detailed shape of the spiral vortex sheet representing the rolled-up shear layer have been presented by Blendermann (1969) and Pullin (1978). It becomes apparent that the initial small time evolution of the vortex is determined through the expansion of (local form of) the outer or generating flow in the vicinity of the sharp edge. For finite but sufficiently high Reynolds numbers, the vortex sheet solutions may then themselves be regarded as an outer inviscid flow which determines the structure of the inner inviscid rotational core and viscous vortex subcore [see Moore \& Saffman (1973) for an analysis of this problem for a related flow and Pullin (1979) for the application of their results to the edge vortex]. After the initial near-edge growth period, the subsequent behaviour of edge vortices and of umbilical shear layers joining them to the forming edge can only be understood within the context of the overall flow.

Starting vortices in a compressible fluid flow have been produced and studied using the method of shock diffraction. Here the interaction of an incident shock wave with a sharp edge at rest with respect to the upstream flow produces a transmitted-reflected wave system bounding an after-flow about the edge which generates the vortex. Rott (1956) showed that for sufficiently weak incident waves the after-flow may be regarded as approximately incompressible near the edge but small density variations still allow the use of interferometry (Howard \& Matthews 1956) or spark shadowgraph (Evans $\&$ Bloor 1977) techniques to visualize the vortex formation process and measure vortex growth rates. Although Evans \& Bloor use an impulsively started model for the afterflow in their vortex discretization calculations of the vortex growth, Rott's weak pulse approximation clearly indicates that a (time $)^{m}$ power law starting flow is more appropriate. The value of $m$ in this special situation depends on the edge angle and is just that required in the general inviscid similarity theory to predict an initial growth rate linear in time. Some workers, however, for example Reichenbach and Merzkirch (1964), have observed an initial nonlinear growth rate preceding an eventual linear rate, which may be due to viscous effects very early in the formation process.

The present experiment was performed with the aim of studying the development of the starting vortex for different edge-forming geometries (wedge angles) and several velocity-time power-law histories of the generating flow. The experiments were carried out in a specially designed water channel in which the starting flow was obtained using a high torque stepping motor controlled through computer software. A ciné film of the dye-in-water patterns produced by the flow was made from which photographic sequences showing the flow development and measurements of the time-wise primary vortex trajectory were obtained. In the following, details of the flow revealed in selected photographic sequences are discussed and the measured vortex centre trajectories are compared with inviscid simliarity theory predictions.

\section{Experimental apparatus}

The experimental apparatus, depicted schematically in figure 1, consisted of a Perspex water channel formed from a tube of rectangular cross-section of height $H=25 \cdot 4$ $\mathrm{cm}$ and width $W=20.3 \mathrm{~cm}$, joined at one end to an open reservoir. At the other end of the tube the flow was driven by a rectangular piston. The water level in the reservoir 


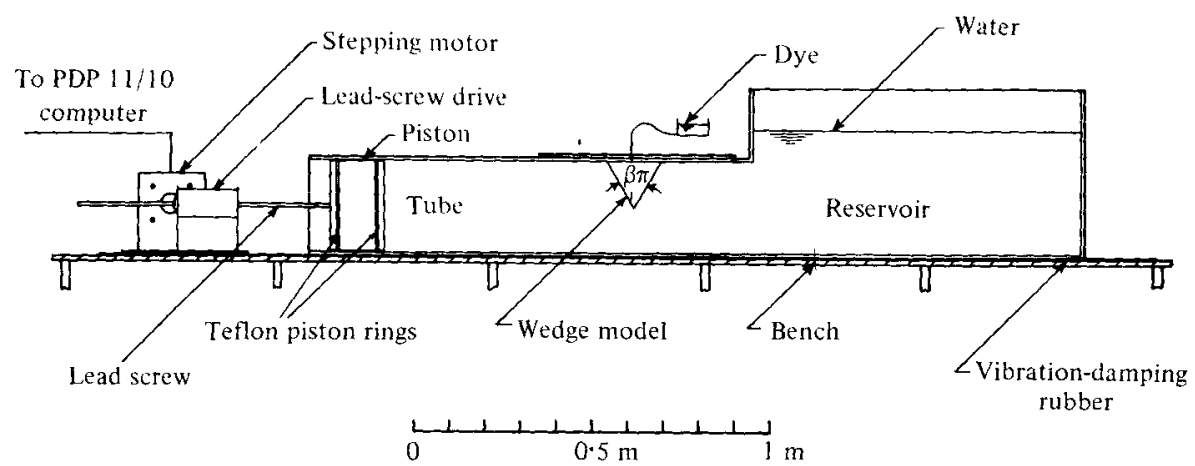

FIGURE 1. Experimental apparatus for starting vortex flow visualization experiment.

always exceeded $H$ so that the tube was always completely full during experimental runs. The wedge model was inserted into the channel through a port and was mounted with the wedge support plate flush with the inner top face of the tube. Each wedge model was in the form of an isosceles triangular prism of height $h=12.70 \mathrm{~cm}$ and length $l=17 \cdot 8 \mathrm{~cm}$. When in position each end of the model was thus separated from the adjacent inner side of the tube by a gap of about $1.25 \mathrm{~cm}$ designed originally to decrease interference between the side-wall boundary layer and the wedge-generated flow. The vortex-forming model edge (wedge apex) of angle $\beta \pi$ radians intersected the tube axis normally $(h / H=0.5)$ and was spaced about $80 \mathrm{~cm}$ from the initial piston position and $30 \mathrm{~cm}$ from the tube-reservoir intersection. In the region of piston movement $W$ and $H$ were toleranced to $0.002 \mathrm{~cm}$. The piston was constructed of Perspex sheeting and was machined to provide a smooth fit inside the tube. It was $15.3 \mathrm{~cm}$ in length and was fitted at each end with Teflon sealing/lubrication rings which provided the only contact with the tube walls. Common grease was used to provide further lubrication.

The piston was driven by a 0.5 in. diameter threaded lead screw with 12 threads/in., which was itself driven through a 1:1 gear box by a high torque stepping motor with a shaft step angle per input drive pulse of $0.9^{\circ}$ (400 steps/revolution). The motor could be operated either at a constant speed via circuitry in the motor drive box or by an externally supplied pulse train. In the present experiment the starting flow was obtained by generating a motor-driving square wave pulse sequence through software on an on-line PDP 11/10 computer. Programmes were written to generate piston velocity-time profiles approximated by

$$
V_{p}=A t^{m},
$$

where $V_{p}$ is the piston velocity, $t$ is the time and $A$ and $m$ are pre-chosen constants. The nominal values of $m$ chosen were $m=0,0 \cdot 25,0.5$ and $1 \cdot 0$. The piston distance-time $(x, t)$ profiles were measured for each $m$ using a video system and are shown in figure 2. It was found that the actual $x, t$ profiles obtained did not exactly correspond to integration of (1) with the prechosen $A, m$, presumably owing to accumulation of small errors. This was not considered important however, and the approximate actual values of $m, A$ achieved were estimated by fitting curves of best least-square fit of the form

$$
x=\frac{A}{1+m} t^{1+m}
$$




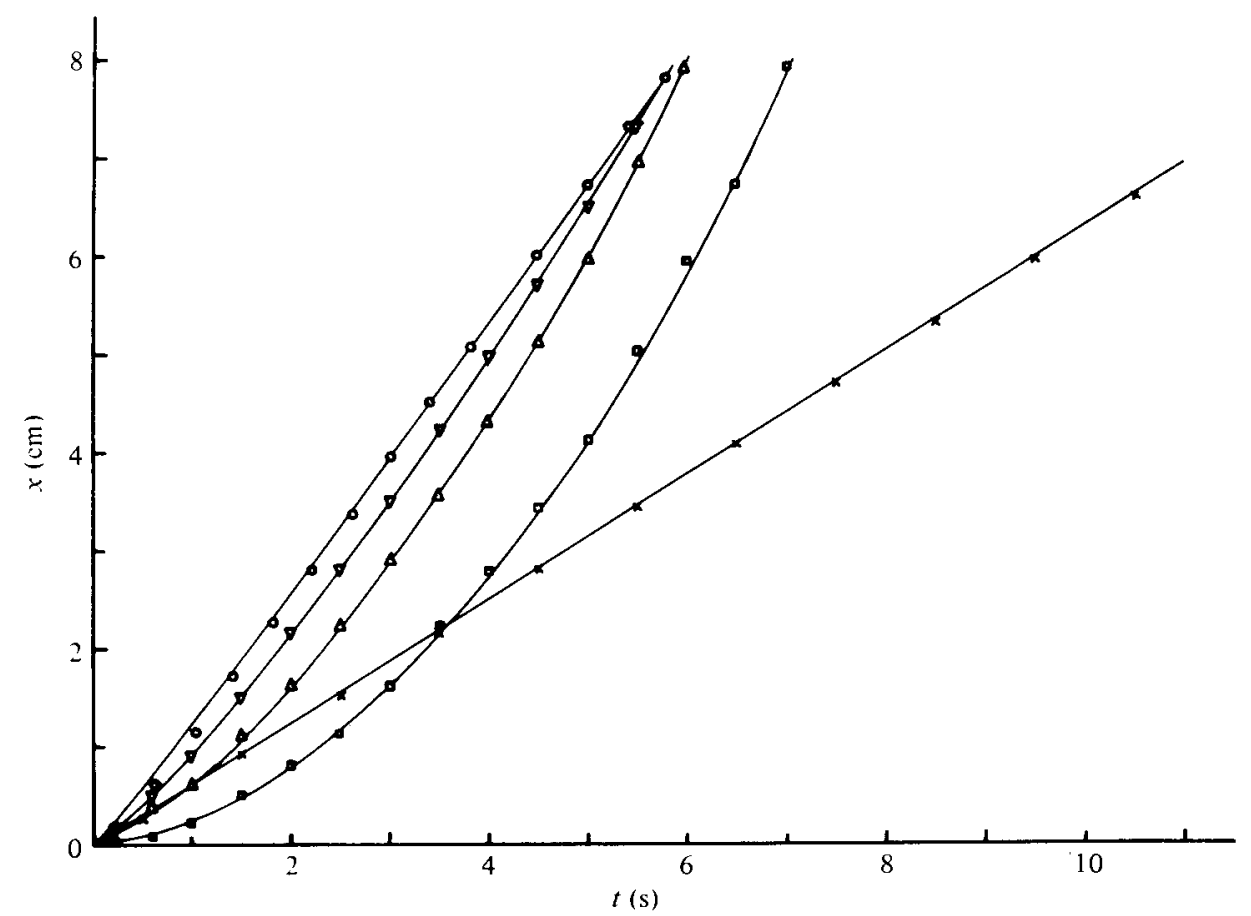

FIGURe 2. Piston position compared with $(\text { time })^{m}$ power laws for parameters $A, m$ given in table $1 ; \times, m=0 ; \odot, m=0.04 ; \nabla, m=0.24 ; \Delta, m=0.45 ; \square, m=0.88$.

\begin{tabular}{|c|c|c|c|}
\hline$m$ & $\begin{array}{c}A \\
\left(\mathrm{~cm} \mathrm{~s}^{-(1+m)}\right)\end{array}$ & $t_{f}(\mathrm{~s})$ & $R e_{c}$ \\
\hline 0 & 0.63 & $12 \cdot 52$ & 1560 \\
\hline $0 \cdot 04$ & $1 \cdot 30$ & $5 \cdot 50$ & 3687 \\
\hline $0 \cdot 24$ & $1 \cdot 10$ & $5 \cdot 68$ & 5109 \\
\hline 0.45 & 0.86 & 5.96 & 6621 \\
\hline 0.88 & $0 \cdot 38$ & $7 \cdot 08$ & 6873 \\
\hline
\end{tabular}

Table 1. Values of time exponent $m$, velocity power law constant $A$, flow times $t_{f}$ and channel Reynolds number $R e_{c}$ for starting flow experiment.

to the measured $x, t$ profiles. Actual values of $A$ and $m$ are given in table 1 together with values of the channel Reynolds number

$$
\operatorname{Re}_{c}=\frac{H^{2}(A / H)^{1 /(1+m)}}{\nu}
$$

In (3) $R e_{c}$ is based on the length and velocity scales $H$ and $H(A / H)^{1 /(1+m)}$ respectively, $\nu$ being the kinematic viscosity of water. In fact none of the values of $m$ shown can be actually achieved owing to infinite accelerations at $t=0$ but the initial time before (2) is approximated is small compared with the duration of the flow. For each case the flow was stopped impulsively at $t=t_{f}$ (see table 1 ).

The flow details were made visible by the use of a readily available household blue dye injected into the water through small holes at various points on the centre-lines of 
each wedge model. A small amount of alcohol was used to make a neutrally buoyant readily visible dye mixture. Dye holes were positioned on both the upstream and downstream faces of each model at distances from the wedge apex which varied with the wedge geometry. It was found by trial and error that careful dye injection before the commencement of each run in conjunction with very slow residual water motions due to previous piston movements induced slow dye movement up the centre-line of the two faces of each wedge, forming thin ribbons along the boundaries at which the shed vorticity forms. In instantaneous snapshots of the subsequent motion the dye trace thus represents flow streaklines. This system, however, did not work well for a thin $5^{\circ}$ wedge model for which the dye holes could be positioned no closer than about $4 \mathrm{~cm}$ from the apex. Hence, when the first dye reached the shedding edge, the vortex was already quite well formed so that the early part of the formation process was not seen. For this model, a very slightly negatively buoyant dye was thus used which initially formed an approximately vertical ribbon as may be seen in the left photo of figure $6(a)$. Instantaneous snapshots then show a combination of streaklines and timelines in the fluid, a timeline being the instantaneous location of a line of particular fluid particles marked (here as the vertical dye ribbon) at a given earlier time. Experience with this dye mixture indicated that the slight negative buoyancy had no observable effect in the time interval $\left(0, t_{f}\right)$ on the flow patterns obtained. The approximate two-dimensionality of the flow near the plane of symmetry near the wedge apex was established by observing dye released through several holes spaced across the wedge model span.

The starting vortices were filmed on $35 \mathrm{~mm}$ black-and-white ciné film at 25 frames $\mathrm{s}^{-1}$. Since this speed is accurately fixed by the camera motor drive, framing may be used as a convenient timing of the flow development. The majority of runs were performed with $5^{\circ}(\beta=0.028)$ and $60^{\circ}(\beta=0.333)$ wedges although some runs were also obtained for a $90^{\circ}(\beta=0 \cdot 5)$ wedge. Two near-impulsive runs, $m=0,0 \cdot 04$, were carried out on the $60^{\circ}$ model to examine two kinematically similar flows at different $R e_{c}$.

\section{Analysis of results}

Two types of results are presented.

(1) Trajectories of the primary 'vortex centre' obtained directly for each run by measurement from frame-by-frame projections of the ciné film.

(2) Photographic sequences comprising prints of selected frames/runs from the ciné film.

Each photographic sequence (figures 5-9) reveals the growth of the primary edge vortex, together with details of various secondary flow phenomena which can be more clearly seen in the selected enlargements of figure 10. The last one or two stills in each sequence show the formation of a vortex pair near the wedge apex after the flow has stopped. These have been included firstly since it was thought that the pair formation process was of some interest in itself as a graphic illustration of Kelvin's theorem, and secondly to enable the reader to compare the single shear layer starting vortex with the double layer stopping vortex. A particularly well-formed vortex pair is depicted in the enlargement of figure 11. As may be seen for example from figure 5, the 'vortex centre' is in general ill defined. The best definition is perhaps the peak of the vorticity distribution in the viscous subcore but since vorticity was not measured we have taken the 


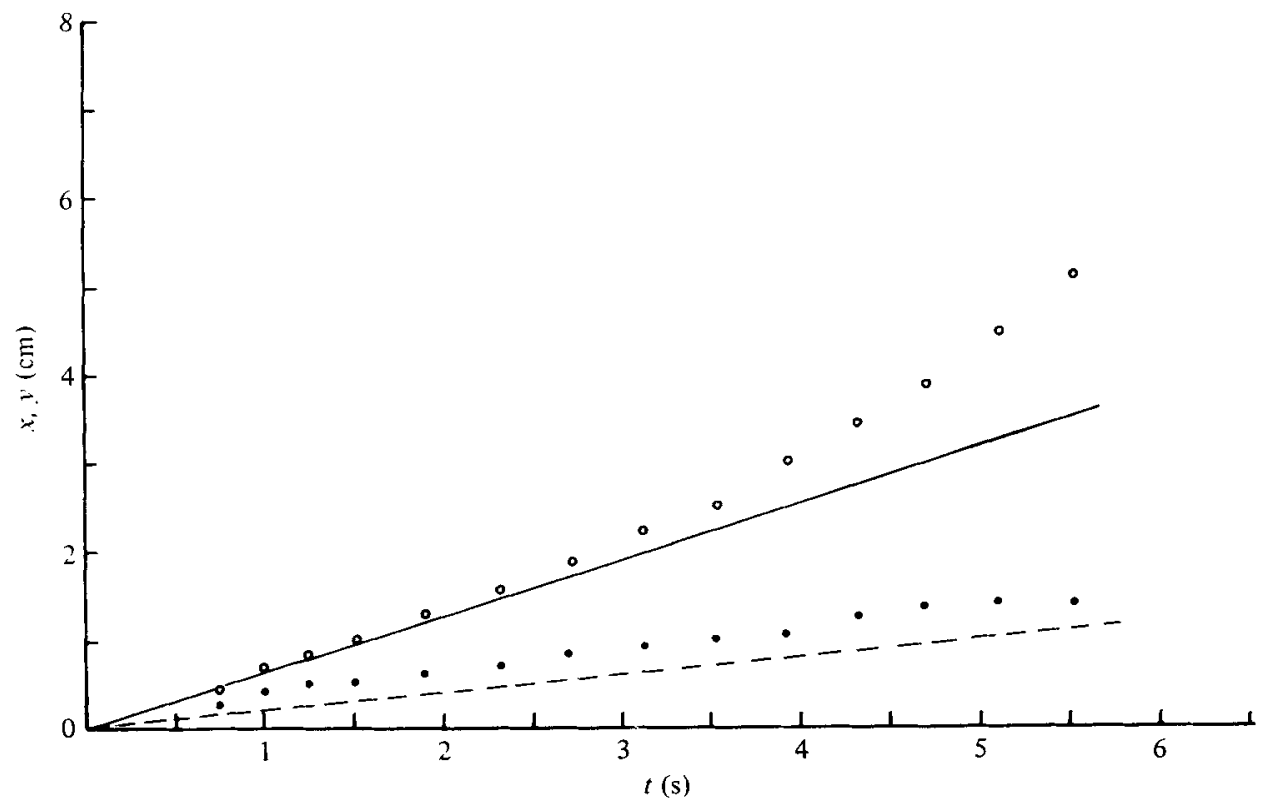

Figure 3. Vertical and horizontal vortex centre positions versus time for $\beta=0.028\left(5^{\circ}\right)$, $m=0 \cdot 45$. Compared with similarity theory predictions. Measurements: $\odot, x ; 0, y$. Theory:

$\longrightarrow, x ;--, y$.

approximate geometric centre, or centre of flow rotation of the last few turns of the vortex streaklines as judged by eye. Figure 3 shows the horizontal $x$ (normal distance from the wedge bisector) and vertical $y$ (distance along wedge bisetrix) vortex centre positions versus time for $\beta=0.028, m=0.45$ compared with similarity theory predictions to be discussed subsequently.

Neglecting three-dimensional flow effects, the infinite-Reynolds-number limit for the starting vortex may be assumed to be that given by the motion of a two-dimensional spiral vortex sheet shed from the wedge apex. For $t$ sufficiently small such that the vortex size is small compared with a typical boundary dimension (say $H$ ), the growth of the vortex sheet should follow similarity laws formulated by Prandtl, Rott (1956) and other workers. Detailed numerical solutions for the self-similar vortex sheet (Pullin 1978) then provide a convenient reference for comparison with the measured vortex trajectories. In these solutions the infinite spiral sheet is represented by a few outer turns adjoining the wedge apex and an isolated vortex representing the overall effect of the inner, tightly rolled-up portion. We shall compare the calculated trajectory of this vortex with the measured vortex centre as described above.

To relate the similarity solutions to the present geometry, we need only expand the outer vortex generating flow in the vicinity of the wedge apex. We assume that a model of the outer flow, appropriate to the channel-wedge geometry, is that of attached twodimensional inviscid incompressible flow down an infinite duct with the wedge positioned at some point on the duct top surface. A similar flow model was employed by Evans $\&$ Bloor (1977) in simulating their shock diffraction flow. For this model with the ductwedge positioned in the $z=x+i y$ plane as in figure 13 , it is shown in the appendix that 
the first two terms in the expansion near the wedge apex $z=0+i 0$, of the complex velocity potential describing the outer flow, are given by

$$
W(z)=-A H t^{m}\left[\alpha_{0}(\beta)\left\{e^{\frac{1}{2} i \pi(3-\beta)}\left(\frac{z}{H}\right)\right\}^{1 /(2-\beta)}+\alpha_{1}(\beta)\left\{e^{\frac{1}{2} i \pi(3-\beta)}\left(\frac{z}{H}\right)\right\}^{3 /(2-\beta)}+\ldots\right],
$$

where $\alpha_{0}(\beta), \alpha_{1}(\beta)$ are dimensionless constants shown plotted versus $\beta$ in figure 14 . The first term in (4) represents a singular attached flow past an effectively infinite wedge while each subsequent term represents a sum of $j$ adjacent nonsingular flows in corners of angle $\pi(2-\beta) / j, j=3,5,7, \ldots$ surrounding the wedge. The weighting of successive terms in (4) by the real constants $\alpha_{0}, \alpha_{1}, \ldots$ accounts for the effect of the duct boundaries so that these constants are determined by the overall duct-wedge geometry. Note that even powers of $1 /(2-\beta)$ do not appear in (4) owing to the antisymmetry of the $y$ velocity component of the flow about $x=0$. The inviscid similarity theory of edge vortex growth follows from the assumption that, for small $t$, the vortex evolution is dominated by the first term in (4). For our purposes it is convenient to write this term in the form

$$
W(z)=-a t^{m} e^{\frac{1}{2} i \pi(3-\beta)(2-\beta)} z^{1 /(2-\beta)},
$$

where

$$
a=\alpha_{0}(\beta) A H^{1-n} \quad \text { with } \quad n=1 /(2-\beta) .
$$

We may now write for the initial trajectory of the vortex centre in a viscous fluid

$$
\begin{aligned}
& x_{v}=x_{v}(a, t, v ; m, n), \\
& y_{v}=y_{v}(a, t, v ; m, n),
\end{aligned}
$$

that is, we consider the generating flow (outer flow) to be inviscid and given by (5), but allow for possible viscous effects in the growing edge vortex (inner flow). Dimensional analysis of (6) then leads to a relationship which we may write as

$$
\begin{aligned}
& \hat{x}_{v} \equiv \frac{x_{v}}{a^{1 /(2-n) t^{M} \mathscr{R}\left[\omega_{v}(m, n)\right]}}=f_{x}\left(R e_{s}\right), \\
& \hat{y}_{v} \equiv \frac{y_{v}}{a^{1 /(2-n)} t^{M \mathscr{I}\left[\omega_{v}(m, n)\right]}}=f_{y}\left(R e_{s}\right),
\end{aligned}
$$

where

$$
R e_{s}=\frac{a^{2 /(2-n) t^{2 M-1}}}{\nu}
$$

with $M=(1+m) /(2-n)$ and where $\mathscr{R}, \mathscr{I}$ represent the real and imaginary parts respectively of a complex argument. In $(7) \omega_{v}(m, n)$ is a complex dimensionless constant which may be obtained from similarity solutions for the edge vortex and $f_{x}, f_{y}$ are unknown functions which may also depend on $m, n$. The scale Reynolds number $R e_{s}$, or non-dimensional time to the power $2 M-1$, is based on internal length scales implicit in (5). It is the natural Reynolds number for the initial growth of the starting vortex and can always be computed provided the near-edge generating flow of the type (4) can be found. For the present outer flow, $R e_{s}$ and $R e_{c}$ are related through

$$
R e_{s}=\alpha_{0}^{2 /(2-n)}\left[(A / H)^{1 /(1+m)} t\right]^{2 M-1} R e_{c} .
$$

Note that equations (7) and (8) may be used to recover the original dimensional results from figures $4(a)-(d)$ which will now be discussed. 

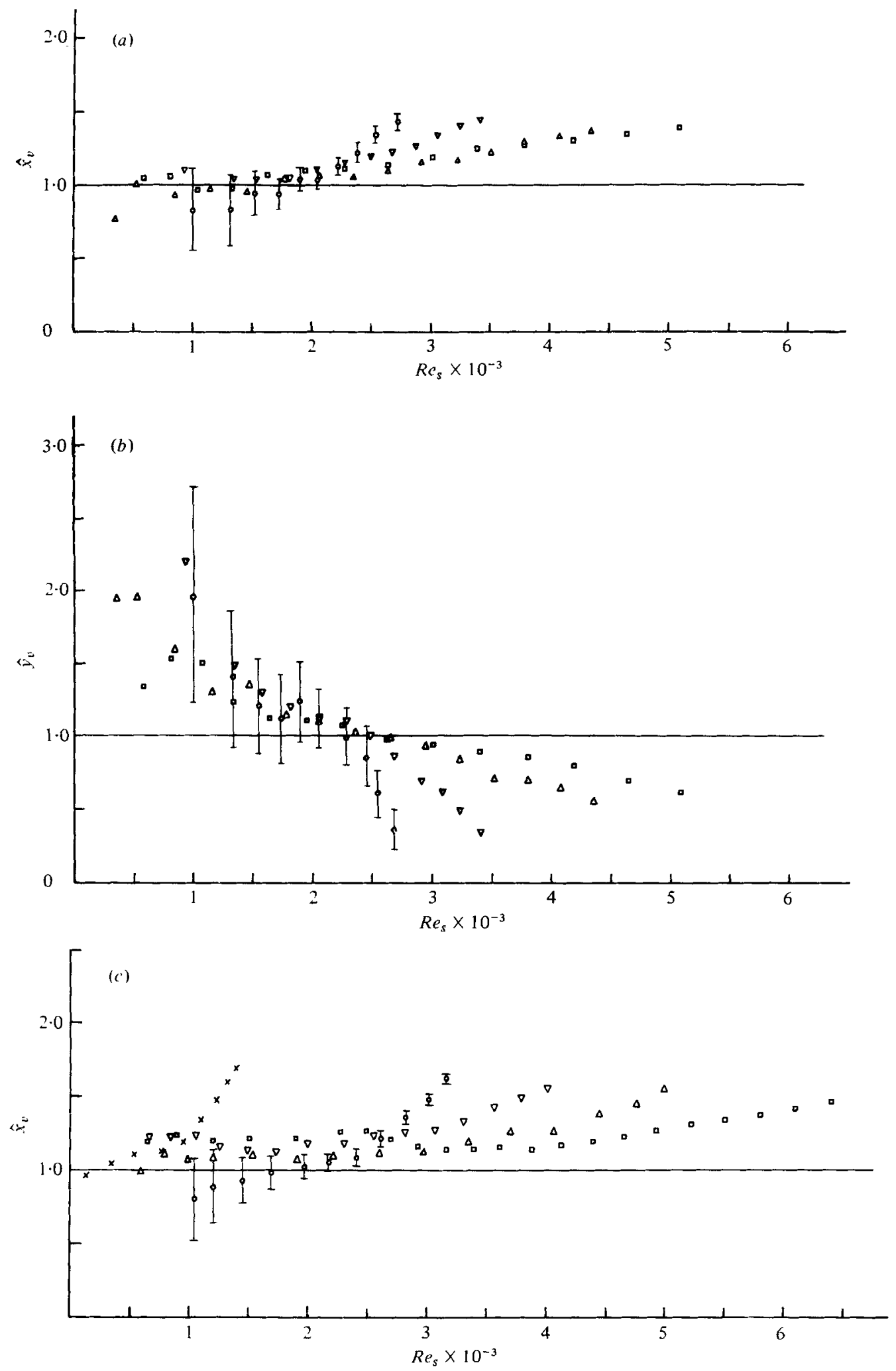

FiaUre 4. For legend see facing page. 

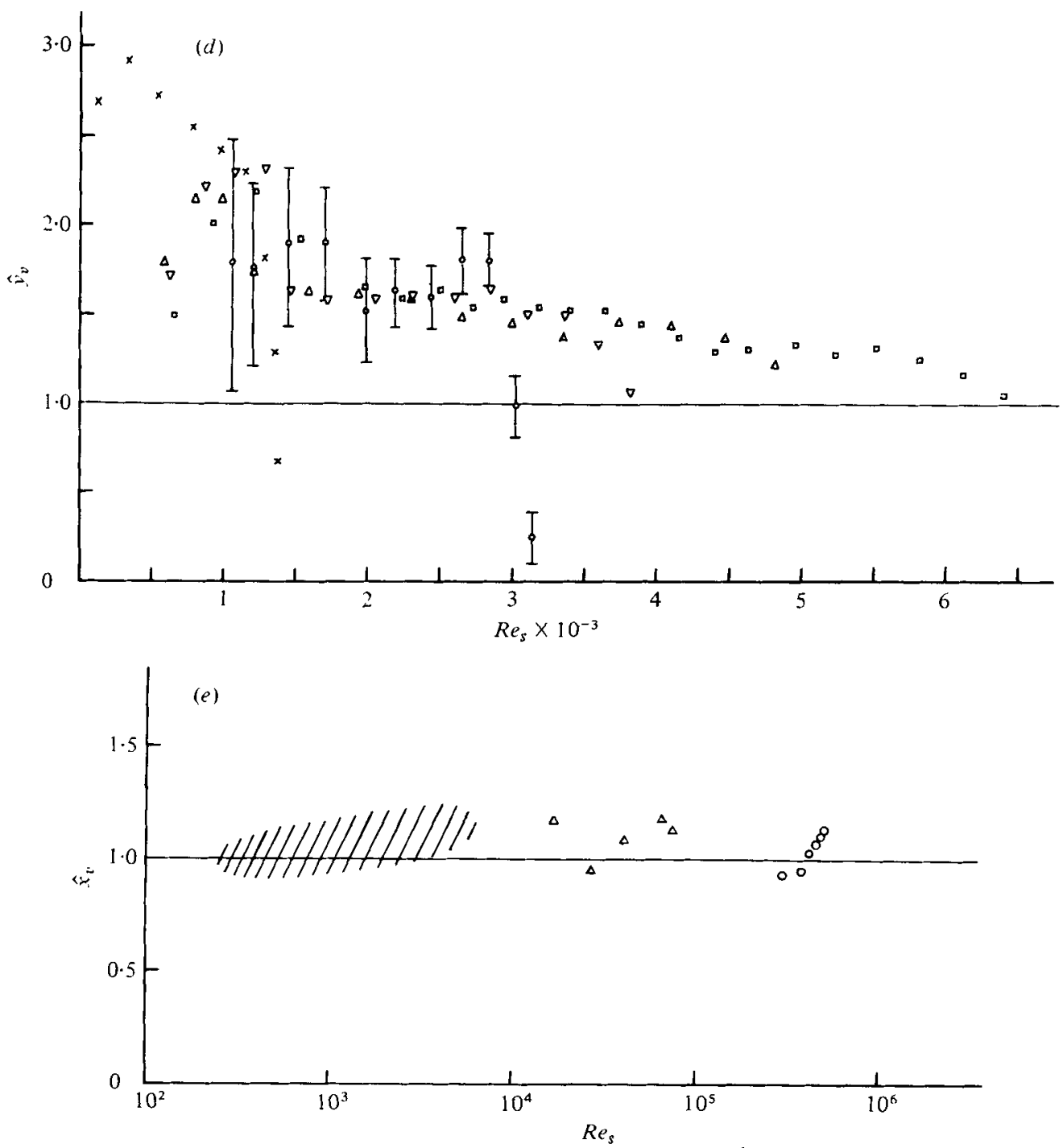

FIGURE 4. Vortex centre positions non-dimensionalized against similarity predictions versus $R e_{s}(a, c, e)$ Horizontal positions. $(b, d)$ Vertical positions. $(a, b) \beta=0.028\left(5^{\circ}\right) . \odot, m=0.04$; $\nabla, m=0.24 ; \Delta, m=0.45 ; \square, m=0.88$. Bars indicate approximate experimental error. $(c, d) \beta=0.333\left(60^{\circ}\right) . \times, m=0 ; \odot, m=0.04 ; \nabla, m=0.24 ; \Delta, m=0.45 ; \square, m=0.88$. (e) $\llbracket$, envelope of present results; $\triangle$, Pierce (1961), $30^{\circ}$ wedge, $m \simeq 1$; $\odot$, Evans \& Bloor (1977), shock diffraction data, $10^{\circ}$ wedge, $m=0$.

\section{Discussion and conclusions}

Measurements of $\hat{x}_{v}$ and $\hat{y}_{v}$ in the form of (7) are shown in figures $4(a-e)$. The values of $\omega_{v}(m, n)$ used given in table 2 were obtained from the calculations of Pullin (1978). For small $t$ and $R e_{s} \rightarrow \infty$ we may assume $f_{x}, f_{y} \rightarrow 1$ so that (7) represents the similarity vortex. Deviations from unity in figures $4(a-e)$ may then be interpreted as either finite $R e_{s}$ effects or the influence of second-order and higher-order terms in (4) distorting the growing vortex, that is, the effect of the channel walls. These two effects are of course 


\begin{tabular}{lllll}
$m$ & \multicolumn{2}{c}{$\beta=0.028$} & \multicolumn{2}{c}{$\beta=0.333$} \\
0 & $\overbrace{\mathscr{R}\left(\omega_{v}\right)}$ & $\mathscr{\mathscr { I } ( \omega _ { v } )}$ & $\overbrace{\mathscr{R}\left(\omega_{v}\right)}$ & $\mathscr{I}\left(\omega_{v}\right)$ \\
0.04 & - & - & 0.318 & 0.060 \\
0.24 & 0.320 & 0.076 & 0.306 & 0.062 \\
0.45 & 0.260 & 0.084 & 0.254 & 0.067 \\
0.88 & 0.220 & 0.084 & 0.217 & 0.066 \\
0.173 & 0.077 & 0.170 & 0.060
\end{tabular}

Table 2. Values of the complex constant $\omega_{v}$ appearing in similarity starting vortex solutions (Pullin 1978).

independent since by moving the piston fast enough (increasing $A$ ) it is possible to generate a very high $R e_{s}$ vortex still close to the wedge apex.

Figures $4(a, c)$ show $\hat{x}_{v}$ versus $R e_{s}$ for the $5^{\circ}$ and $60^{\circ}$ wedges respectively. Although estimates of the experimental error for $R e_{s} \lesssim 2000$ are of the same order as the differences between theory and experiment the consistent trend indicates that measurement exceeds theory by some $10-20 \%$ at low $R e_{s}$ for the $60^{\circ}$ wedge. For $\hat{y}_{v}$ in figures $4(b, d)$ the disagreement is of order $50-100 \%$ but here relative experimental errors are large since $y_{v}$ itself is rather smaller than $x_{v}$. At low $R e_{s}$ these discrepancies can be attributed to finite $R e_{s}$ or viscous effects, notably:

(1) The displacement effect on the primary edge vortex of the secondary vortex which forms owing to boundary-layer separation on the leeward wedge surface between the moving pressure minima induced by the primary vortex and the wedge apex (see Smith 1966 for a detailed discussion of this effect for the related problem of leadingedge separation from a slender delta wing). For the present flow, the extent of this vortex can be clearly seen in figure 5 (plate 1 ) and figure $10 \alpha(\beta=0.333, m=0)$, figure 6 (plates 2 and 3$)(\beta=0 \cdot 333, m=0 \cdot 45$, right sequences), figure 7 (plate 4 ) $(\beta=0.333, m=0.04)$ and figure 8 (plate 5$)(\beta=0.333, m=0.88)$. It is just visible in figures $9(d-f)$ (plate 6$)(\beta=0 \cdot 5, m=0 \cdot 24)$ but can be seen more clearly for this flow in figure $10(b)$ (plate 7$)$. The secondary vortex does not appear in figure $6(\beta=0.028$, $m=0 \cdot 45$, left-hand sequence) probably because the induced pressure minimum is sufficiently close to the wedge apex to inhibit its formation. This may explain the superior agreement of $x_{v}$ with the similarity theory in figure $4(a)$ for the $5^{\circ}$ wedge. (See also figure 11 , plate 8 .)

(2) The possibly related phenomenon of a near-apex bubble, that is, a region near the wedge apex in which the streamlines form closed loops on a much smaller scale than that of the primary vortex. This bubble can be seen clearly in figures 5, 7 and 9 (see figure 10 for selected enlargements) and in all cases it appears to be about the same size as the secondary vortex region. The bubble is not visible in the $m=0.45$, 0.88 flows of figures 6 and 8 (even though quite strong secondary separation is apparent for $\beta=0 \cdot 33$ ) so that it may be associated with near-impulsive flows. A similar phenomenon has been observed by Thompson (1975) who used the hydrogen bubble technique to view steady flow leading-edge vortex formation on a slender wing of rhombic cross-section at Reynolds numbers of order $10^{4}$ based on the wing centre-line chord. Thompson considers this feature to be a kind of tertiary vortex rotating in the same sense as the primary vortex and induced by the secondary separation vortex. It seems likely that this bubble lies entirely within the shear layer separating from the sharp 
edge and may perhaps be seen as a region of approximately constant vorticity near the wedge apex. It does not appear in the higher $R e_{s}$ experiments of Pierce (1961) but this may be due to the flow visualization method used or to the fairly slender wedges employed.

(3) The effects of finite shear-layer thickness. Simple diffusion arguments may be used to show that the ratio of the shear-layer thickness just prior to separation at the wedge apex to the overall vortex size is given by $K R e_{s}^{-\frac{1}{2}}$. A very rough estimate of $K$ for a particular case, namely $m=1-n$, indicates that $K \simeq 5$ so that for the present flows the near-apex shear layer is relatively thick. Since the concept of the separated structure as a tightly wound spiral vortex sheet in an otherwise irrotational flow then becomes suspect, certainly on the scale of the shear-layer thickness, the reasonable quantitive agreement of the low $R e_{s}$ results with infinite $R e_{s}$ theory is unexpected. It seems that potential flow theory works quite well provided that the correct circulation distributions 'in the large' are maintained.

At higher $R e_{s}, \hat{x}_{v}$, shows a systematic deviation from the similarity theory which can be seen clearly in figure 3 and which is also evident in the photo sequences. In figure 5 for example, the vortex is approximately the same shape but on a different scale in figures $5(a-d)$. In figures $5(e-f)$ it does not increase greatly in size (the rolling-up process has ceased) but movement away from the wedge is evident. This break-down of similarity behaviour can be attributed to the influence of the channel boundaries and to three-dimensional flow effects. While the latter are difficult to evaluate, it is clear that channel boundary effects can alone lead to deviation from similarity when the first term in (4) no longer dominates the near-apex attached flow velocity field so that second and subsequent terms begin to influence (in a very complicated way) the vortex evolution. An estimate of this effect may be obtained by examining the relative magnitude of the first two terms in the expansion of $d W / d z$ near the wedge apex. From (4) it follows that the ratio $\Delta$ of the magnitude of the complex velocity due to the second term to that due to the first singular term is $\Delta=3 \alpha_{1} / \alpha_{0}(|z| / H)^{2 /(2-\beta)}$. Using values of $\alpha_{0}, \alpha_{1}$ given in figure 14 we find that for $\beta=0 \cdot 028, \Delta=0 \cdot 15$ for $|z| / H \simeq 0 \cdot 10$, increasing to $\Delta=0.3$ at $|z| / H \simeq 0.20$. From figure 3 it may be seen that these values of $|z| / H$ roughly cover the range of $\left|z_{v}\right| / H$ over which significant deviation from similarity begins to occur.

Figure $4(e)$ compares the envelope of the present $\hat{x}_{v}$ with the much higher $R e_{s}$ results of Pierce (1961) and Evans \& Bloor (1977). Those of Pierce are only for small time where the velocity profile of his moving plate conforms to $m \simeq 1$. While we feel that Evans $\&$ Bloor's case corresponds more closely to an $m=0.5$ flow, they have been reduced as $m=0$ as they were presented originally in this way. In figure 12 the similarity theory predictions of the vortex sheet shape for two cases are compared with experimental dye streakline patterns corresponding to the photographs of figure 10. Even though $R e_{s}$ is relatively low in both cases the experimental and theoretical streaklines are quite similar. On the experimental facsimile we have superimposed separatrices showing suggested critical point patterns (see Perry \& Fairlie 1974) for instantaneous streamlines. Note in each case the saddle point at which the three closed streamline regions (primary and secondary vortex and leading-edge bubble) and the outer flow meet. In cases where the bubble does not appear, perhaps at higher $R e_{s}$, this full saddle point might well degenerate into a half-saddle separating the primary and secondary vortex regions. 


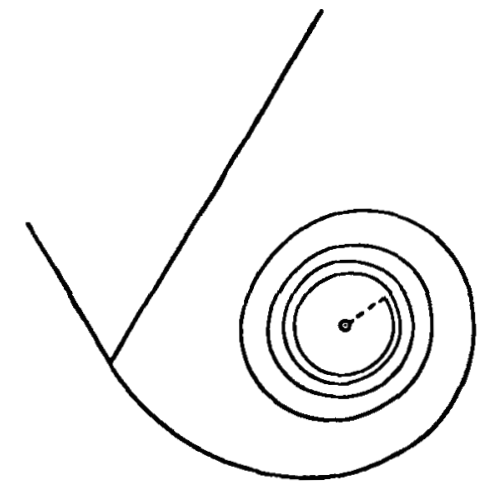

(i)

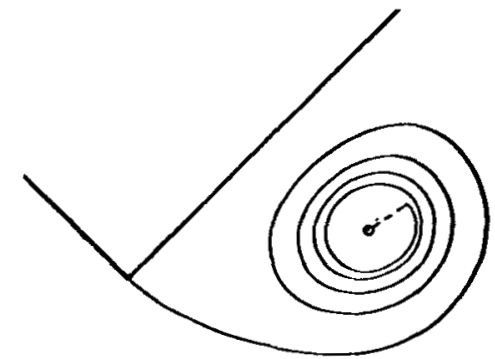

(i)

(b)

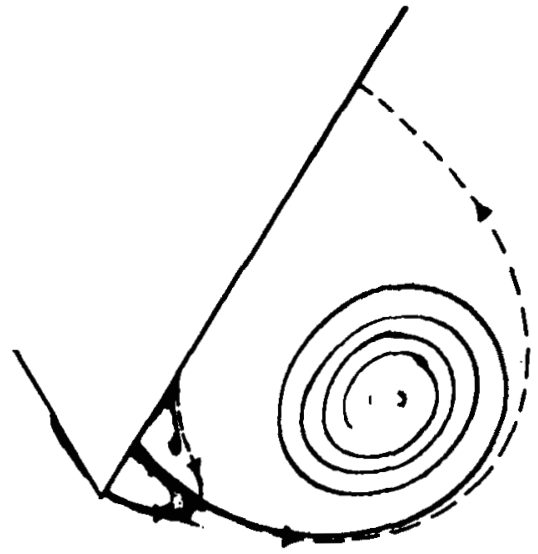

(ii)

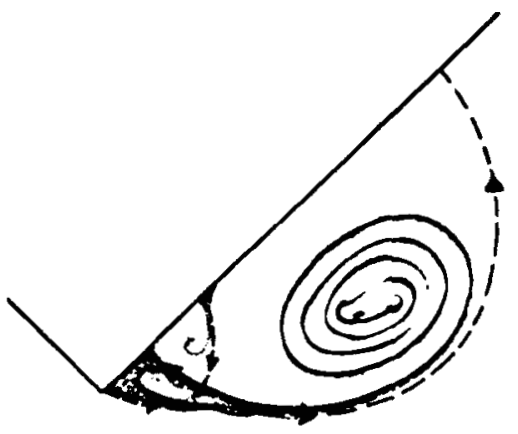

(ii)

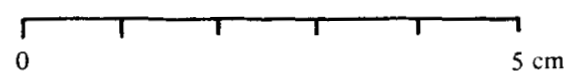

FIGURe 12. Comparison of (ii) experimental and (i) similarity theory predictions of streaklines produced in a starting vortex. (a) $\beta=0.333\left(60^{\circ}\right), m=0, t=5 \mathrm{~s}, R e_{s}=967$. (b) $\beta=0.5$ $\left(90^{\circ}\right), m=0 \cdot 25, t=4 \mathrm{~s}, R e_{s}=3385$. Dashed lines are separatrices showing suggested critical point patterns for instantaneous streamlines. A separatrix is an instantaneous streamline joining the saddle-points.

Several workers (e.g. Howard \& Matthews (1956), Pierce (1961)) have observed the formation of apparent rotation centres at small angular intervals along the rolled-up shear layer in the starting vortex. Indeed these 'small vortices' are the most salient feature of Pierce's well-known spark shadowgraph photos. Professor P. T. Fink (private communication) has also observed a similar phenomenon in unpublished photographs of impulsively started flow past a flat plate, but found that it vanished when certain sources of apparatus vibration were eliminated. The phenomenon is clearly visible in the left-hand sequence of figure $6(\beta=0 \cdot 028, m=0 \cdot 45)$ and photographic sequences of other $\beta=0.028$ flows not presented here indicate that it was generally present to a greater or lesser degree for this geometry. The phenomenon is suggested by the formation of kinks in the streakline patterns in the $\beta=0 \cdot 333$ 
sequence of figure 6 and also in figure 7 , but is not visible in the lowest $R e_{c}$ sequence of figure 5, or in those of figure 9. Evidence that this effect must be associated with apparatus vibration in the present experiment is provided by a comparison of figures $5(d-e)$ with figures $7(a-b)$. These photo pairs correspond to similar values of $\beta, m$ and $R e_{s}$ and therefore to a similar near-edge unperturbed flow, but to different values of $A$, and thus to different external vibration levels.

Although we believe that the level of apparatus vibrations in the present experiments was low by most standards (no formal measurements were taken) vibration was inevitably present to some extent, the most likely source being within the stepping motor lead/screw drive coupling mechanism. The frequency range of $1-5 \mathrm{kHz}$ associated with the step-wise operation of the stepping motor is perhaps too high to be responsible for readily observable unsteady periodic phenomena in the fluid. Although solid friction 'stiction' effects were initially present in the piston-tube contact, these were subsequently reduced by loosening the fit of the piston in the flume, and by careful lubrication of contact surfaces. Since the wedge models were not supported at their sides but only along the tube top surface, apparatus vibration seems most likely to have been transmitted to the shear layer through the thin $5^{\circ}$, wedge, a conclusion which appears to be supported by our photographic evidence.

The question then remains as to whether the streakline behaviour apparent in figure 6 for $\beta=0.028$ actually represents the formation of 'discrete vortices' within the shear layer as a manifestation of the stability properties of the layer. A strong case for extreme caution in such an interpretation has been presented by Hama (1962). Hama has shown that streaklines in a 'tanh-type' shear-layer velocity profile perturbed by an unamplified travelling sinusoidal-velocity wave may have the appearance of amplification and roll up as if to indicate the formation of discrete vortices, when in fact no such roll-up or vorticity concentrations are present. Computed critical layer (that level in the shear layer where the mean fluid velocity and the wave propagation speed are equal) streaklines shown in figures 2-4 of Hama's paper bear a marked resemblance to the pattern of streaklines from the $5^{\circ}$ wedge apex in figure 6 and also to those in Pierce's (1961) photographs. The appearance of these patterns then does not necessarily indicate a shear-layer instability but may be interpreted alternatively as complex streakline behaviour in an apparatus-induced neutrally perturbed flow.

The analysis of Moore (1975) indicates that the infinite $R e_{s}$ asymptotic form of the spiral vortex sheet generated by a starting flow is stable to small two-dimensional disturbances. The stabilizing mechanism absent in plane shear layers is stretching of the vortex sheet along its length due to the local strain field applied by the overall flow. The relevance of this analysis to the presentflows is somewhat doubtful however, since the perhaps important effects of finite shear-layer thickness and local compression of the vortex sheet along its outer turns (indicated by the calculations of Pullin (1978)) are not included. We nevertheless conclude that Hama's (1962) interpretation of the streakline behaviour might be considered as an alternative to the stability interpretation thus indicating that the edge-generated shear layer may be stable in the large, at least in the sense that it does not tend to break up into 'discrete vortices'. Future investigations of finite shear-layer stability which include forcing function effects may throw further light on this question. 

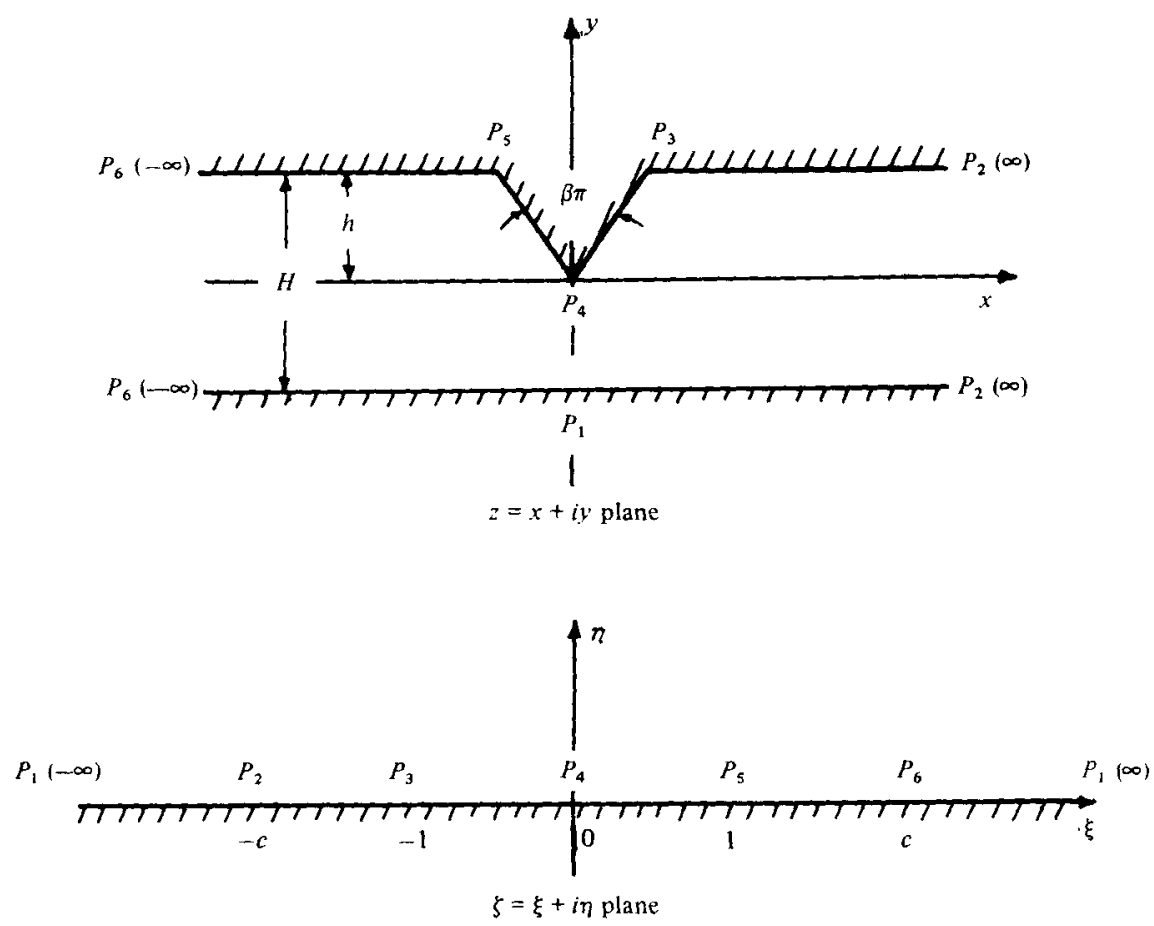

FrguRE 13. Mapping of infinite duct and wedge in $z=x+i y$ plane to upper half $\zeta=\xi+i \eta$ plane.

\section{Note added in revision}

A referee has pointed out that there is an apparent periodicity associated with $y_{v}$ in figure 3 . Re-examination of our raw data showed that a similar periodicity may have been present for other cases, positive identification being rather difficult owing to large relative experimental errors for this quantity. If real, we can offer no suitable explanation for this phenomenon. It may be also attributed to apparatus vibration, but this seems unlikely.

\section{Appendix. Expansion of the channel flow near the wedge apex}

The interior of the region in the $z$ plane bounded by the walls of the infinite twodimensional duct and the wedge surface, denoted by $P_{1} P_{2} P_{3} P_{4} P_{5} P_{6}$ in figure 13, may be mapped into the upper half $\zeta=\xi+i \eta$ plane through the Schwarz-Christoffel transformation

$$
z=K \int_{0}^{\zeta} \frac{\zeta^{\prime 1-\beta} d \zeta^{\prime}}{\left(\zeta^{\prime 2}-c^{2}\right)\left(\zeta^{\prime 2}-1\right)^{\frac{1}{(1-\beta)}}}
$$

where $K$ and $c$ are a complex and a real positive constant respectively. Choosing suitable branches for the fractional powers in (9) and applying this transformation to $P_{4} P_{1}$ and $P_{4} P_{5}$ respectively in the $z$ plane leads to

$$
\begin{aligned}
H-h & =K \int_{0}^{\infty} \frac{\eta^{1-\beta} d \eta}{\left(\eta^{2}+c^{2}\right)\left(\eta^{2}+1\right)^{\frac{1}{2}(1-\beta)}}, \\
h & =K \cos \left(\frac{\beta \pi}{2}\right) \int_{0}^{1} \frac{\xi^{1-\beta} d \xi}{\left(c^{2}-\xi^{2}\right)\left(1-\xi^{2}\right)^{\frac{1}{(1-\beta)}}},
\end{aligned}
$$


which are sufficient to determine $K$ and $c^{2}$. Putting $\delta=h / H$, expressing the integral in $(0, \infty)$ in $(10 a)$ in terms of that in $(0,1)$ in $(10 b)$ by integration around an appropriate contour in the $\zeta$ plane, and eliminating $K$ yields an equation for $c^{2}$ as

$$
\frac{1}{\delta} \cos \left(\frac{\pi \beta}{2}\right) \int_{0}^{1} \frac{\xi^{1-\beta} d \xi}{\left(c^{2}-\xi^{2}\right)\left(1-\xi^{2}\right)^{\frac{1}{2}(1-\beta)}}-\frac{\pi}{2} c^{-\beta}\left(c^{2}-1\right)^{(\beta-1) / 2}=0 .
$$

For $\beta=0$ (11) has the solution $c^{2}=1+\cot ^{2}\left(\frac{1}{2} \delta \pi\right)$. For $\beta>0$ numerical solutions to (11) were obtained using a Newton-Raphson scheme in conjunction with 24 point Gaussian quadrature to evaluate a suitably transformed version of the integral. From $(10 b)$ and (11), $K$ may then be obtained as

$$
\frac{K}{H}=\left(\frac{2}{\pi}\right) c^{\beta}\left(c^{2}-1\right)^{\frac{1}{2}(1-\beta)} .
$$

The complex velocity potential in the $\zeta$ plane corresponding to flow in the positive $x$ direction in the $z$ plane channel is

$$
W(\zeta)=\frac{H A t^{m}}{\pi} \log \left(\frac{\zeta-c}{\zeta+c}\right)
$$

corresponding to a source of strength $2 H A t^{m}$ at $\zeta=c$ and a sink of strength $2 H A t^{m}$ at $\zeta=-c$. The expansion of the attached flow near the wedge apex may now be obtained by combining the expansions of $(9)$ and (13) near $\zeta=0$ to yield

$$
W(z)=-H A t^{m} \sum_{j=0}^{\infty} \alpha_{j}(\beta)\left\{e^{\frac{1}{2} i \pi(3-\beta)}\left(\frac{z}{H}\right)\right\}^{(2 j+1) /(2-\beta)},
$$

where the $\alpha_{j}$ are known functions of $K / H$ and $\beta$. Using (12) $\alpha_{0}$ and $\alpha_{1}$ may be obtained as

$$
\begin{gathered}
\alpha_{0}(\beta)=\frac{2}{\pi}\left\{\frac{\pi(2-\beta)}{\left.2\left(c^{2}-1\right)^{\frac{1}{2(1-\beta)}}\right\}^{1 /(2-\beta)}}\right. \\
\alpha_{1}(\beta)=\frac{\pi^{2}(1-\beta)\left(1-\frac{3}{2} c^{2}\right)}{12(4-\beta)} \alpha_{0}^{3}(\beta)
\end{gathered}
$$

Figure 14 shows the variation of $\alpha_{0}$ and $\alpha_{1}$ with $\beta$ using values of $c^{2}(\beta)$ obtained from the numerical solutions of (11) for $\delta=\frac{1}{2}$, which is appropriate to the present geometry. Note that, as $\beta \rightarrow 1, c \rightarrow 1, \alpha_{0} \rightarrow \infty$. 


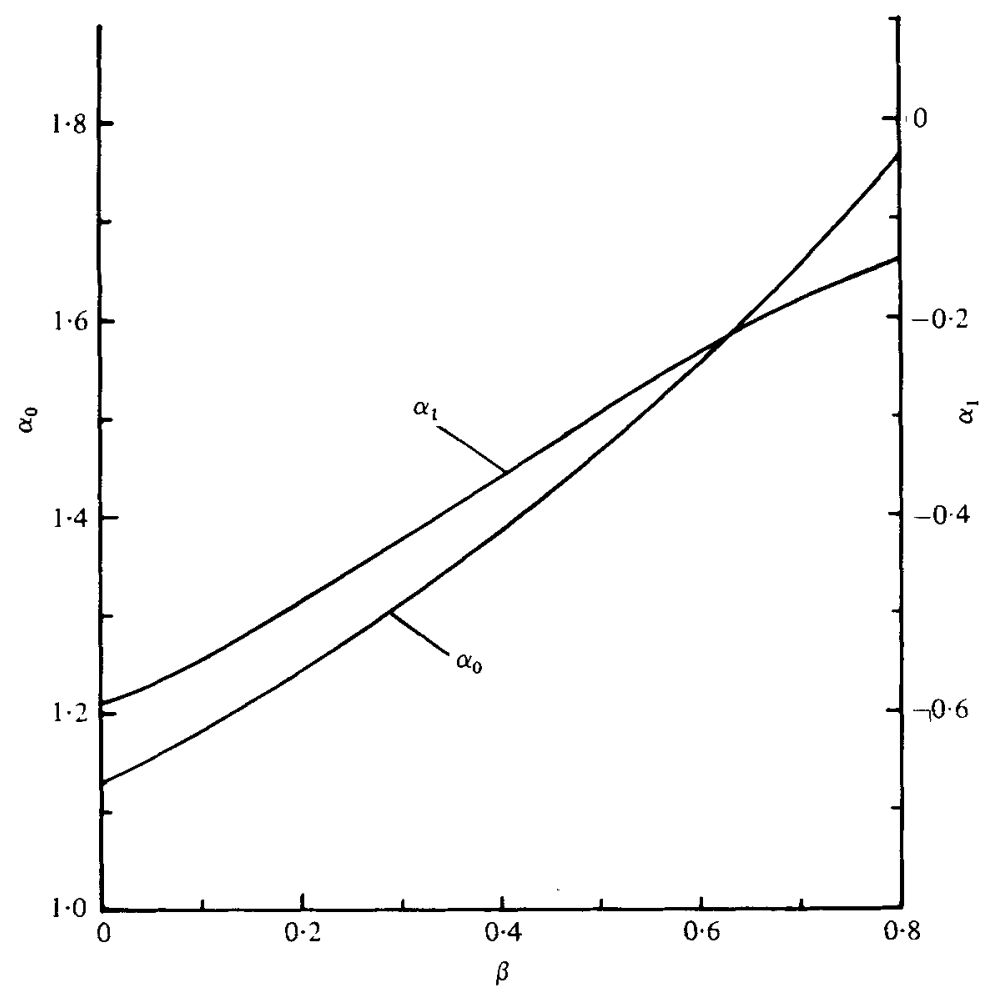

FIGURE 14. Variation with $\beta$ of constants $\alpha_{0}, \alpha_{1}$, in expansion of complex potential near wedge apex.

The authors would like to thank Dr M. S. Chong who wrote the Fortran programmes for the PDP 11/10 and also Mr D. Gardner who very ably handled the photography.

This work was supported by the Australian Research Grants Committee.

\section{REFERENCES}

BAtChelor, G. K. 1970 An Introduction to Fluid Dynamics. Cambridge University Press.

BlendermanN, W. 1969 Der Spiral-Wirbel am translatorisch bewegten Kreisbogenprofil; Structur, Bewegung und Reaktion. Schiffstechnik, 16, 3-14.

Evans, R. A. \& Bloor, M. I. G. 1977 The starting mechanism of wave-induced flow through a sharp edged orifice. J. Fluid Mech. 82, 115-128.

Hama, F. R. 1962 Streaklines in a perturbed shear flow. Phys. Fluids 5, 644-650.

Howard, L. N. \& MatThews, D. L. 1956 On the vortices produced in shock diffraction. J. Appl. Phys. 27, 223-231.

Moore, D. W. 1975 The stability of an evolving two-dimensional vortex sheet. Mathematika $23,128-133$.

Moore, D. W. \& Saffman, P. G. 1973 Axial flow in laminar trailing vortices. Proc. Roy. Soc. A 333, 491-508.

Perry, A. E. \& Fairife, B. D. 1974 Critical points in flow patterns. Adv. Geophys. 18, 299-315.

PIERCE, D. 1961 Photographic evidence of the formation and growth of vorticity behind plates accelerated from rest in still air. $J$. Fluid Mech. 11, 460-464.

PuLIIN, D. I. 1978 The large-scale structure of unsteady self-similar rolled-up vortex sheets. J. Fluid Mech. 88, 401-430. 
Pulln, D. I. 1979 Vortex formation at tube and orifice openings. Phys. Fluids 22, 401-403.

ReICHENBACH, H. \& MERzkrRCH, W. 1964 Untersuchungen über das Ähnlichkeitsverhalten einer interstationaren Wirbelspirale. $Z$. Flugwiss. 12, 219-221.

RotT, N. 1956 Diffraction of a weak shock with vortex generation. J. Fluid Mech. 1, 111-128.

Saffmand, P. G. 1978 The number of waves on unstable vortex rings. J. Fluid Mech. 84, 625-639.

Sмгтн, J.H. B, 1966 Improved calculations of leading-edge separation from slender delta wings. R.A.E. Tech. Rep. no. 66070.

Thompson, D. H. 1975 A water tunnel study of vortex breakdown over wings with highly swept edges. Australian Defence Scientific Service Aeronaut. Res. Lab. Aerodyn. Note 356.

Wrdemeyer, E. 1956 Ausbildung eines Wirbelpaares an den Kanten einer Platte. MaxPlanck-Institut für Strömungsforschung, Göttingen, Bericht 56/B/06. 


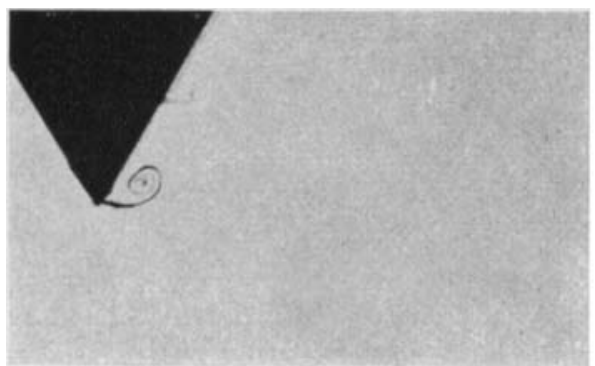

(a)

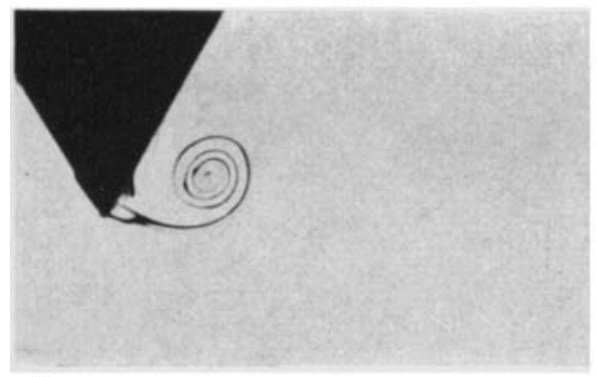

(b)

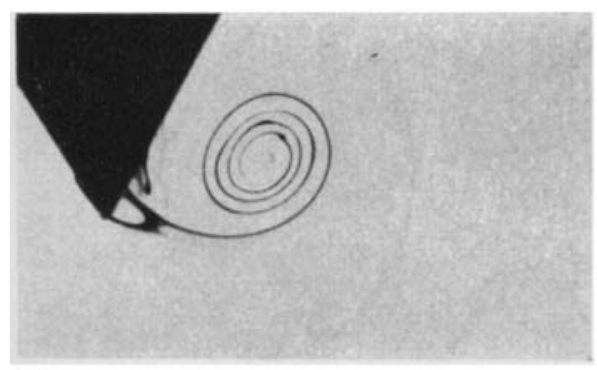

(c)

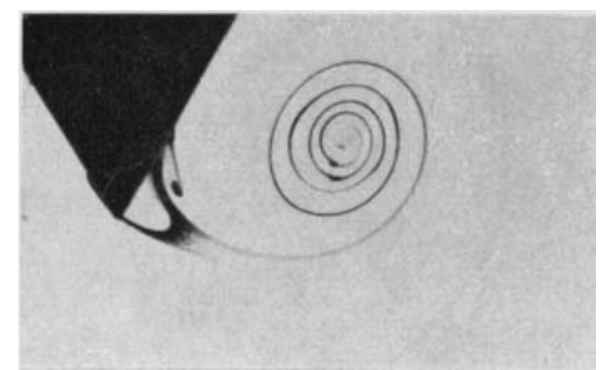

(d)

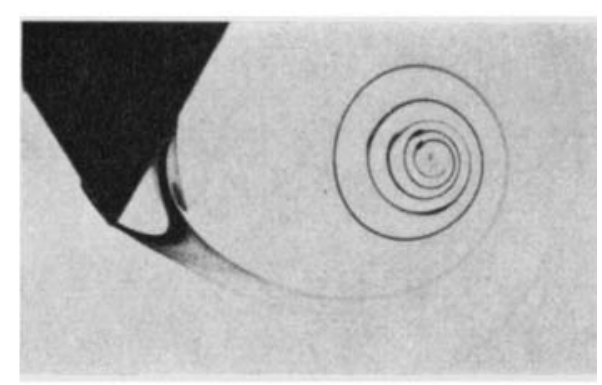

(e)

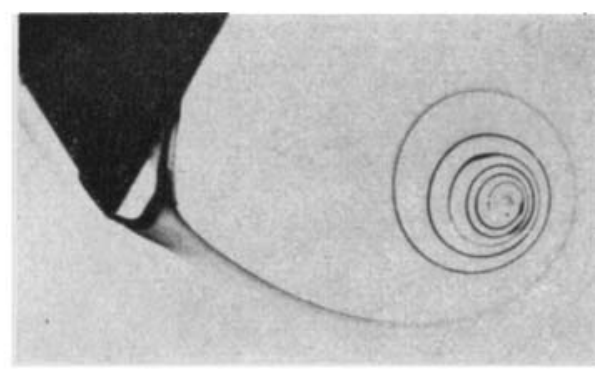

$(f)$

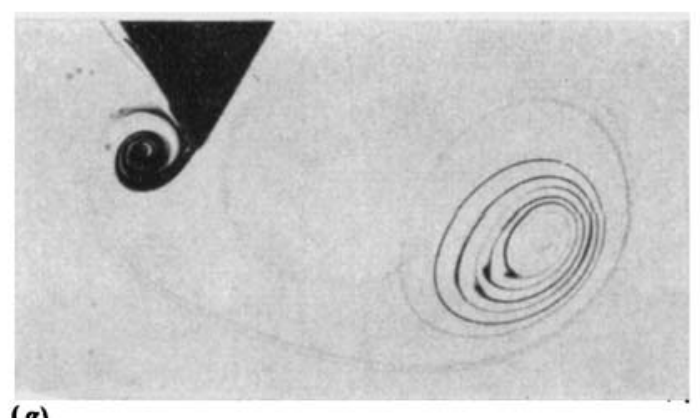

$(g)$

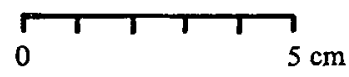

Figure 5. Sequence of photos showing dye released from the surface of a wedge into a startingstopping flow in water $\beta=0.333\left(60^{\circ}\right), m=0, R e_{c}=1560$. The flow stopped at $t=12.52 \mathrm{~s}$. (a) $t=1.0 \mathrm{~s}, R e_{\mathrm{s}}=485 ;(b) t=3.0 \mathrm{~s}, R e_{s}=776 ;(c) t=5.0 \mathrm{~s}, R e_{s}=967 ;(d) t=7.0 \mathrm{~s}, R e_{s}=$ $1117 ;(e) t=9.0 \mathrm{~s}, R e_{s}=1244 ;(f) t=11.0 \mathrm{~s}, R e_{s}=1356 ;(f) t=13.0 \mathrm{~s}$. 
(a)

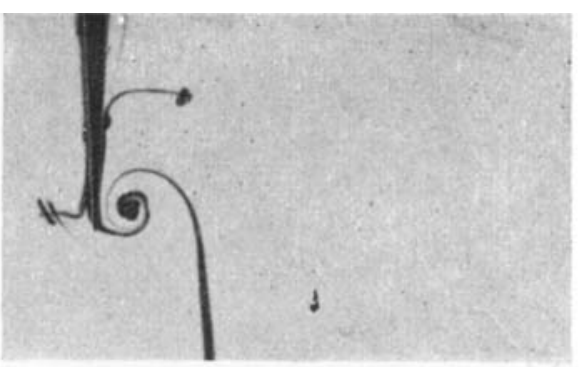

(i) $t=1.0 \mathrm{~s}, R e_{s}=843$

(b)

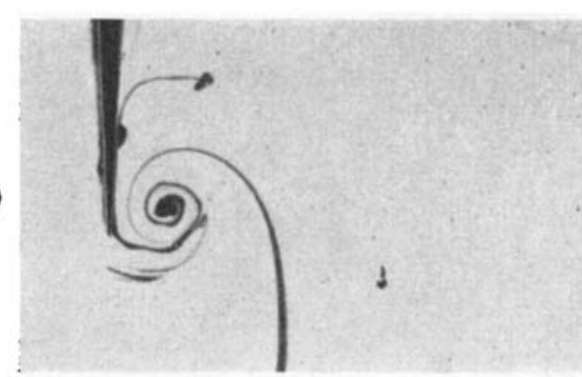

(i) $t=1.6 \mathrm{~s}, k e_{s}=1308$

(c)

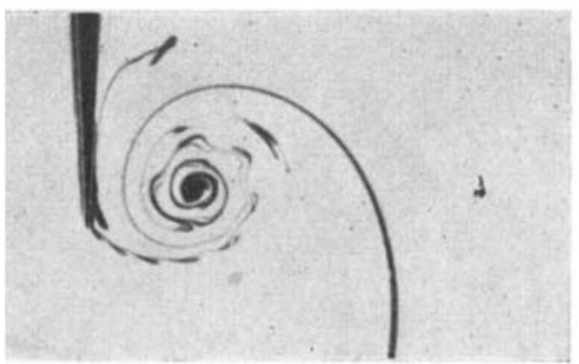

(i) $t=2.8 \mathrm{~s}, R e_{s}=2205$

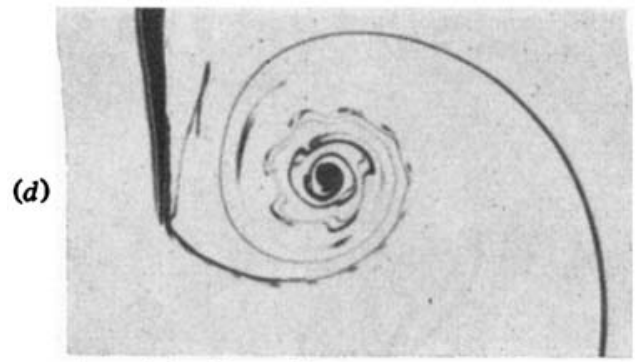

(i) $t=4.0 \mathrm{~s}, R e_{s}=3076$

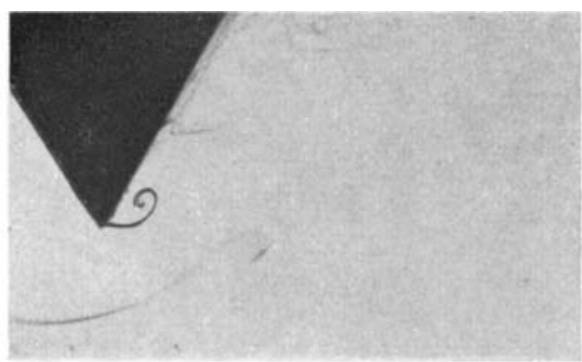

(ii) $t=1.0 \mathrm{~s}, R e_{s}=774$

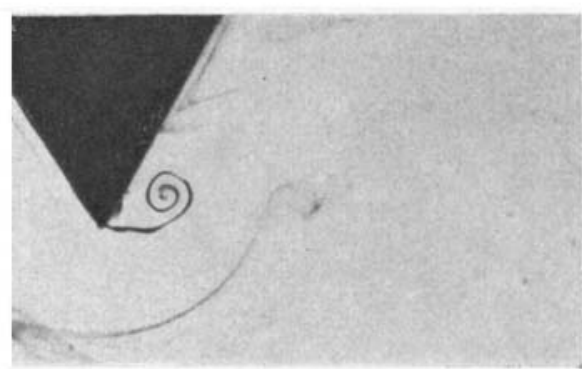

(ii) $t=1.6 \mathrm{~s}, R e_{s}=1280$

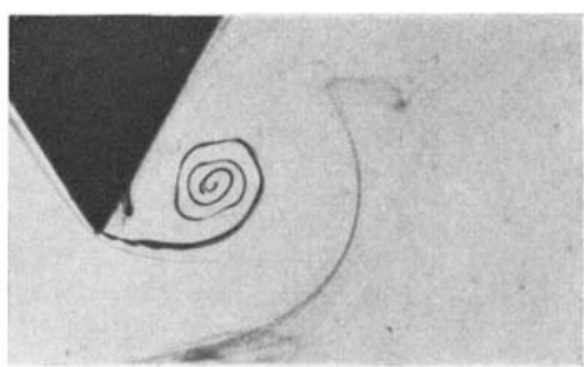

(ii) $t=2.8 \mathrm{~s}, R e_{s}=2333$

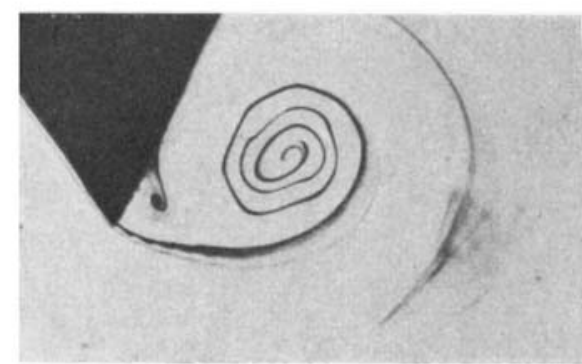

(ii) $t=4.0 \mathrm{~s}, R e_{s}=3418$

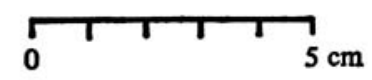

Figure 6. See plate 3 for the legend.

PULLIN AND PERRY 
(e)

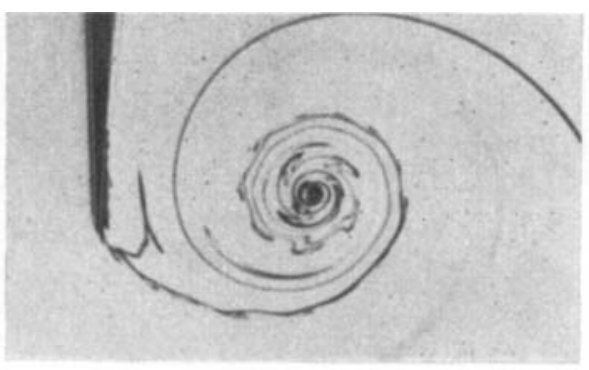

(i) $t=5.0 \mathrm{~s}, R e_{s}=3789$

${ }_{1}(f)$

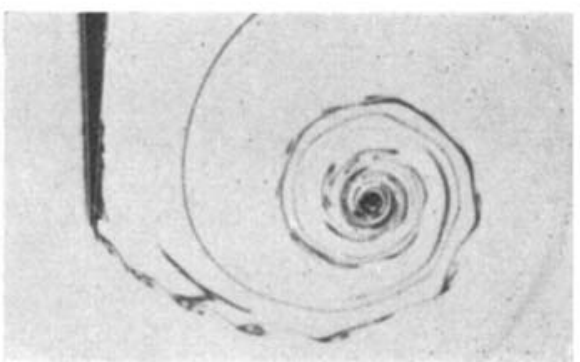

(i) $t=5.68 \mathrm{~s}, R e_{t}=4268$

(g)

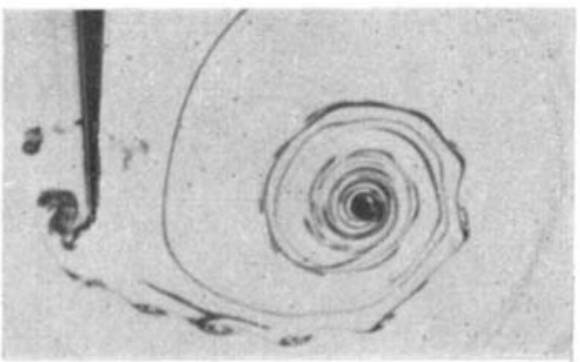

(i) $t=6.08 \mathrm{~s}$

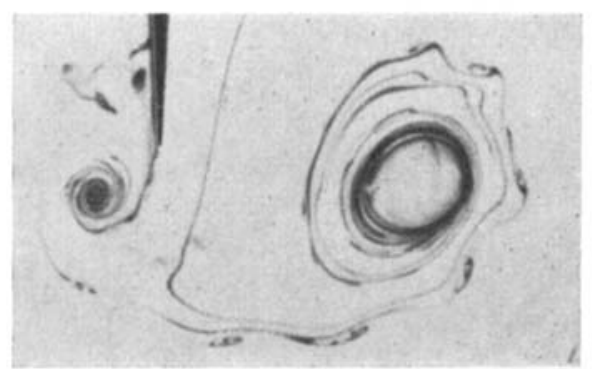

(i) $t=6.30 \mathrm{~s}$

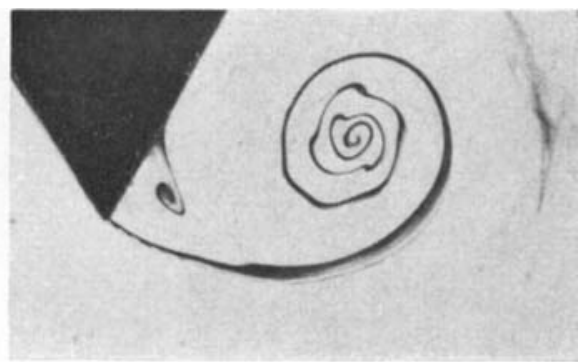

(ii) $t=5.0 \mathrm{~s}, R e_{s}=4342$

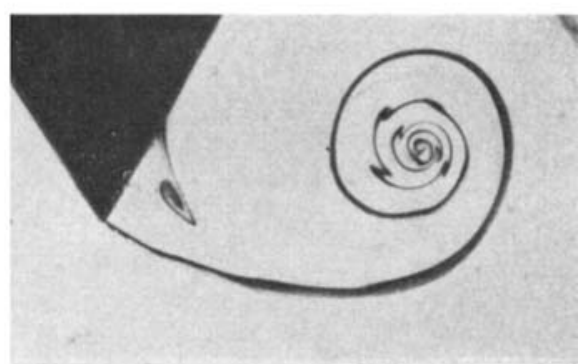

(ii) $t=5.68 \mathrm{~s}, R e_{s}=4978$

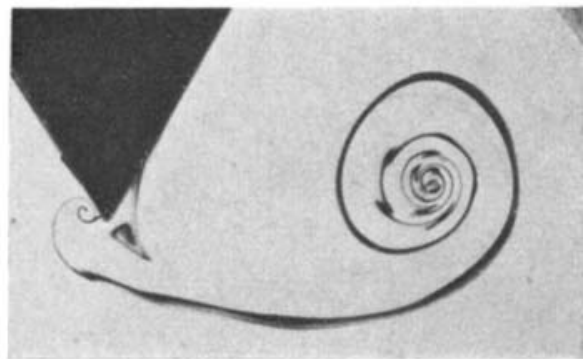

(ii) $t=6.08 \mathrm{~s}$

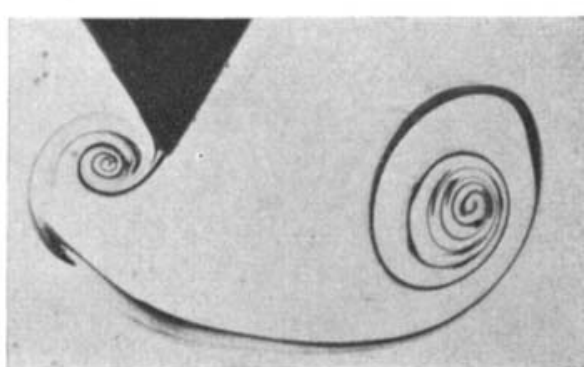

(ii) $t=6.80 \mathrm{~s}$

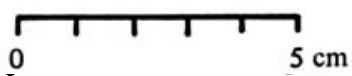

Figure 6. Comparison of two photo sequences showing dye released from the surface of a wedge into a starting-stopping flow in water. The sequences correspond to the same outer flow with $m=0.45, R e_{c}=6621$ but different wedge angles (i) $\beta=0.028\left(5^{\circ}\right)$, (ii) $\beta=0.333\left(60^{\circ}\right)$. Times $t$ after start of flow and $R e_{s}$ are shown. Flow stops at $t=5.96 \mathrm{~s}$. Note in the left-hand sequence the presence of both streaklines from the wedge apex and smoother timelines (see $\$ 2$ for definition) may be seen in the fluid. 


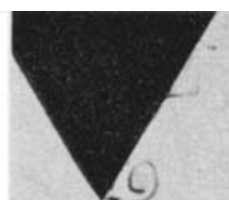

(a)

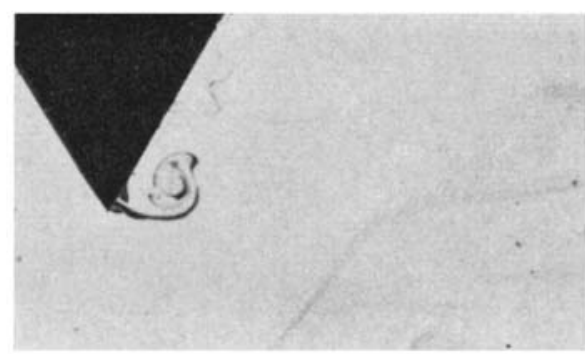

(b)

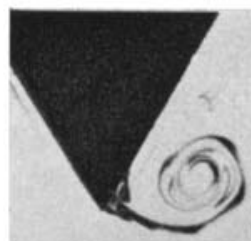

(c)

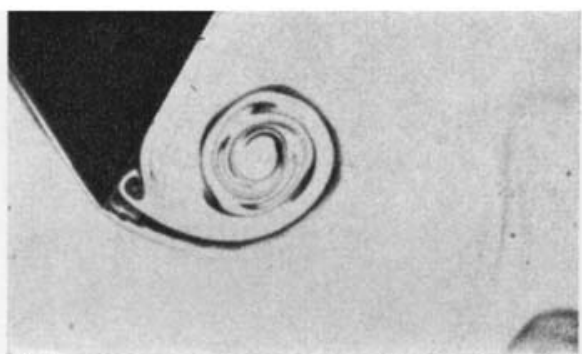

(d)

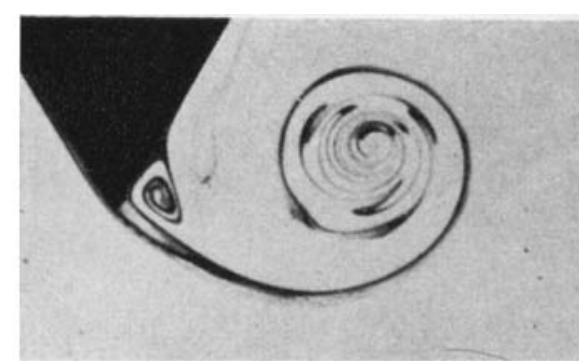

(e)

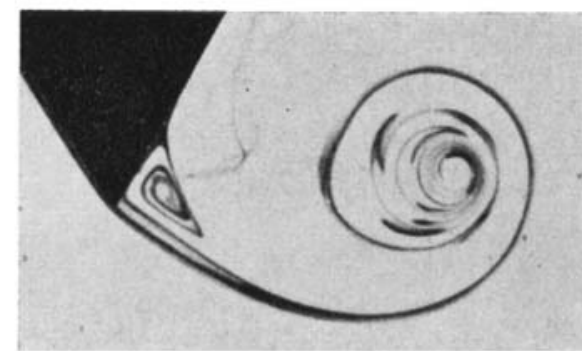

$(f)$

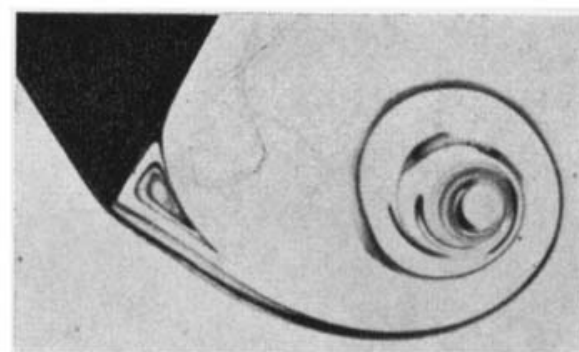

(

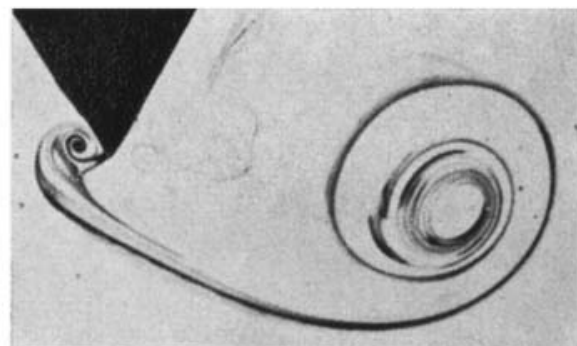

$($ ( $)$

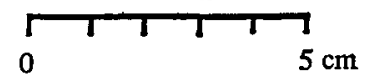

Figure 7. Sequence of photos showing dye released from the surface of a wedge into a startingstopping flow in water, $\beta=0.333\left(60^{\circ}\right), m \approx 0.04, R e_{c}=3687$. Flow stops at $t=5 \cdot 50 \mathrm{~s}$. $(a)$ $t=0.6 \mathrm{~s}, R e_{s}=1090 ;(b) t=1.0 \mathrm{~s}, R e_{s}=1397 ;$ (c) $t=1.6 \mathrm{~s}, R e_{s}=1744 ;(d) t=2.8 \mathrm{~s}, R e_{s}=$ $2302 ;(e) t=4.0 \mathrm{~s}, R e_{s}=2739 ;(f) t=4.8 \mathrm{~s}, R e_{s}=2993 ;(g) t=5.28 \mathrm{~s}, R e_{s}=3135 ;(h) t=$ $6 \cdot 0 \mathrm{~s}$.

PULLIN AND PERRY 
Journal of Fluid Mechanics, Vol. 97, part 2

Plate 5

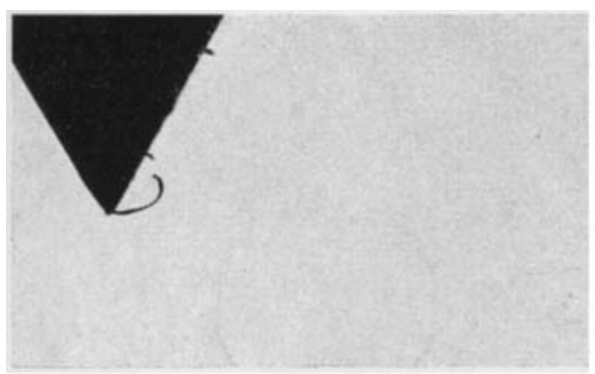

(a)

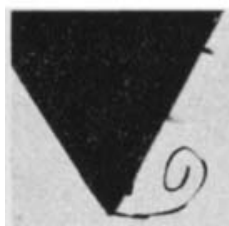

(b)

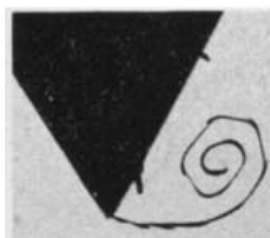

(c)

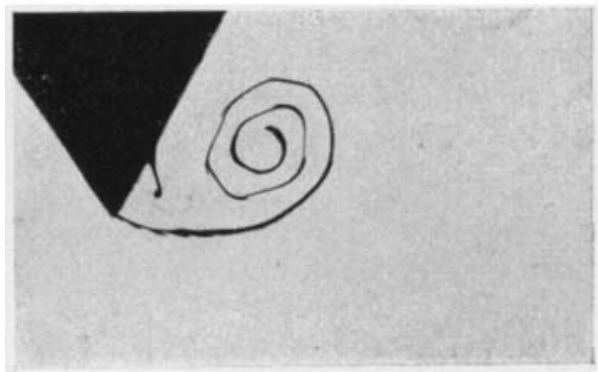

(d)

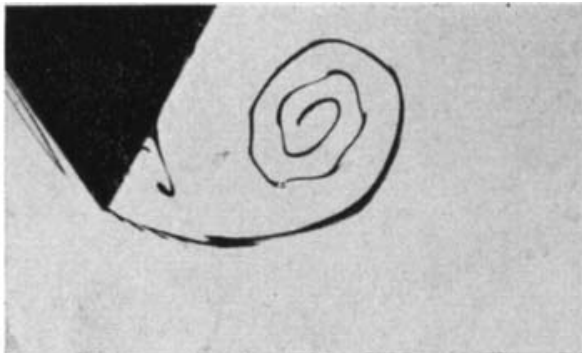

(e)

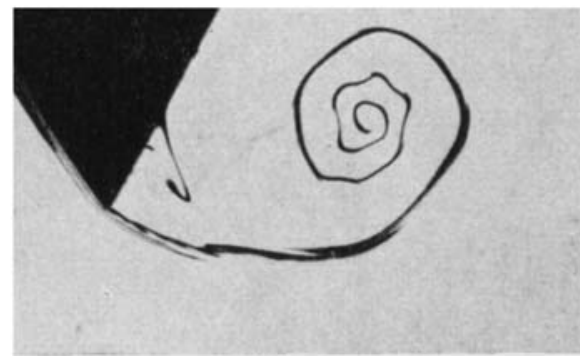

(f)

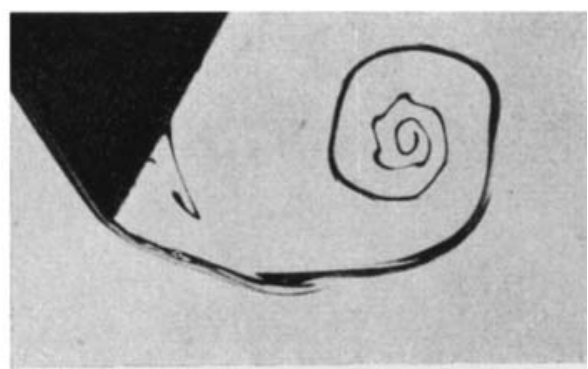

(g)

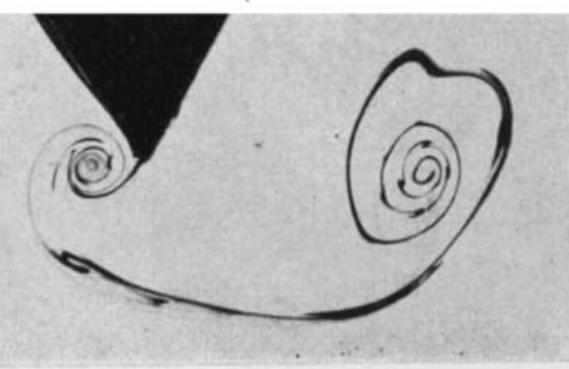

(h)

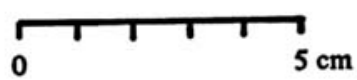

FigURE 8. Sequence of photos showing dye released from the surface of a wedge into a startingstopping flow in water, $\beta=0.333\left(60^{\circ}\right), m=0.88, R e_{c}=6873$. Flow stops at $t=7.08 \mathrm{~s} .(a) t=$ $1.8 \mathrm{~s}, R e_{s}=649 ;(b) t=2.8 \mathrm{~s}, R e_{s}=1367 ;(c) t=4.0 \mathrm{~s}, R e_{s}=2455 ;(d) t=5.0 \mathrm{~s}, R e_{s}=3634$ (e) $t=6.0 \mathrm{~s}, R e_{\mathrm{s}}=4941 ;(f) t=6.6 \mathrm{~s} ; R e_{s}=5802 ;(g) t=7.0 \mathrm{~s}, R e_{s}=6408 ;(h) t=7.6 \mathrm{~s}$. 


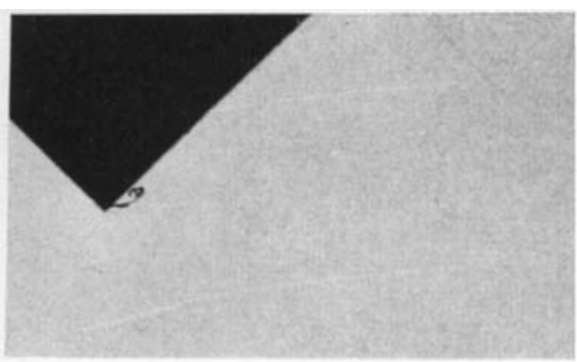

(a)

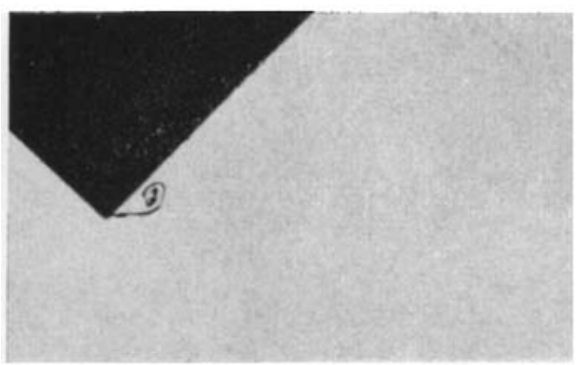

(b)

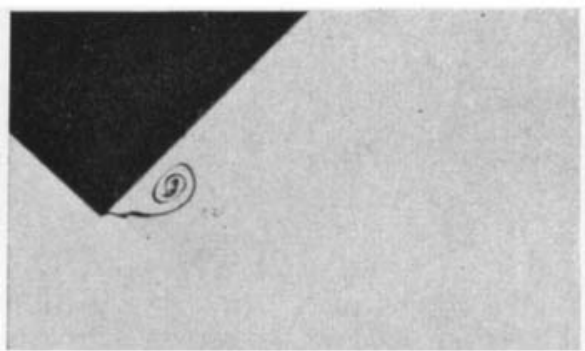

(c)

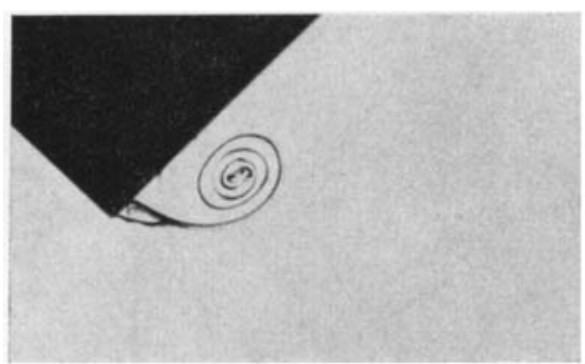

(d)

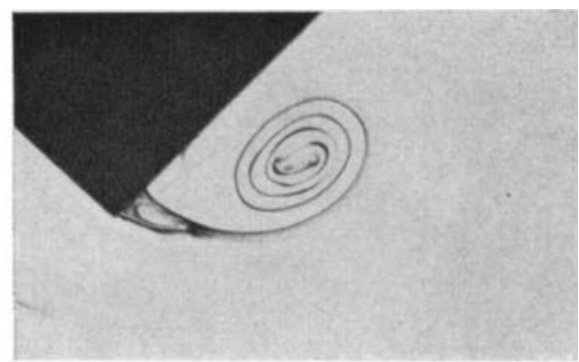

(e)

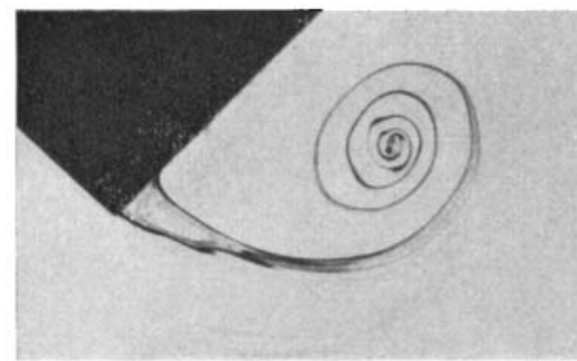

(f)

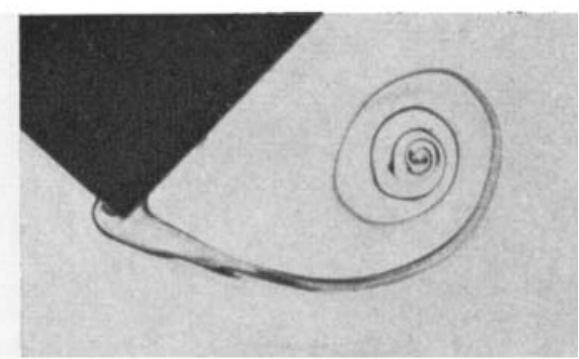

(g)

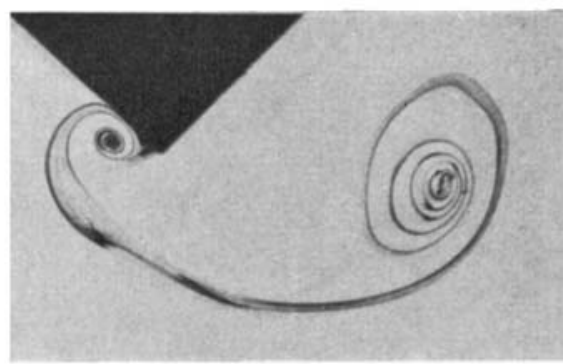

(h)

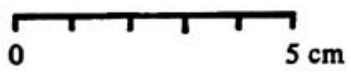

FxGURe 9. Sequence of photos showing dye released from the surface of a wedge into a startingstopping flow in water, $\beta=\frac{1}{2}\left(90^{\circ}\right), m=0.24, R e_{c}=5109$. Flow stops at $t=5 \cdot 68 \mathrm{~s} .(a) t=0.6 \mathrm{~s}$, $R e_{s}=662 ;(b) t=1.0 \mathrm{~s}, R e_{s}=1027 ;(c) t=1.6 \mathrm{~s}, R e_{s}=1539 ;(d) t=2.8 \mathrm{~s}, R e_{s}=2490 ;$ (e) $t=4.0 \mathrm{~s}, R e_{s}=3385 ;(f) t=5.28 \mathrm{~s}, R e_{s}=4298 ;(g) t=5.88 \mathrm{~s} ;(h) t=6.40 \mathrm{~s}$. 


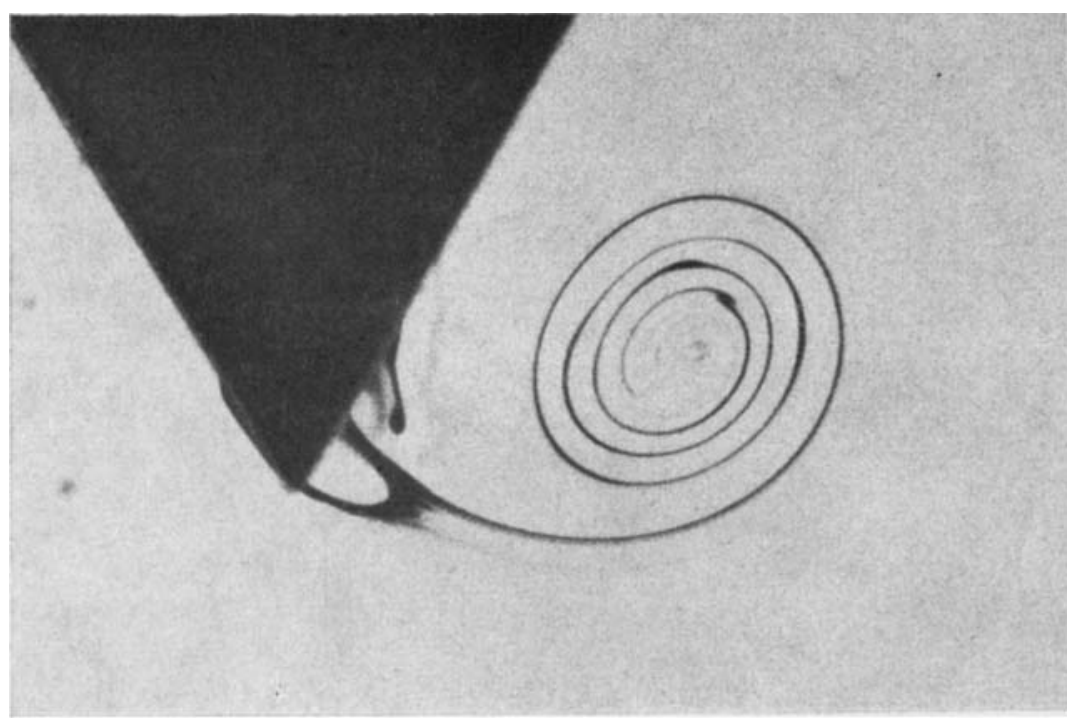

(a)

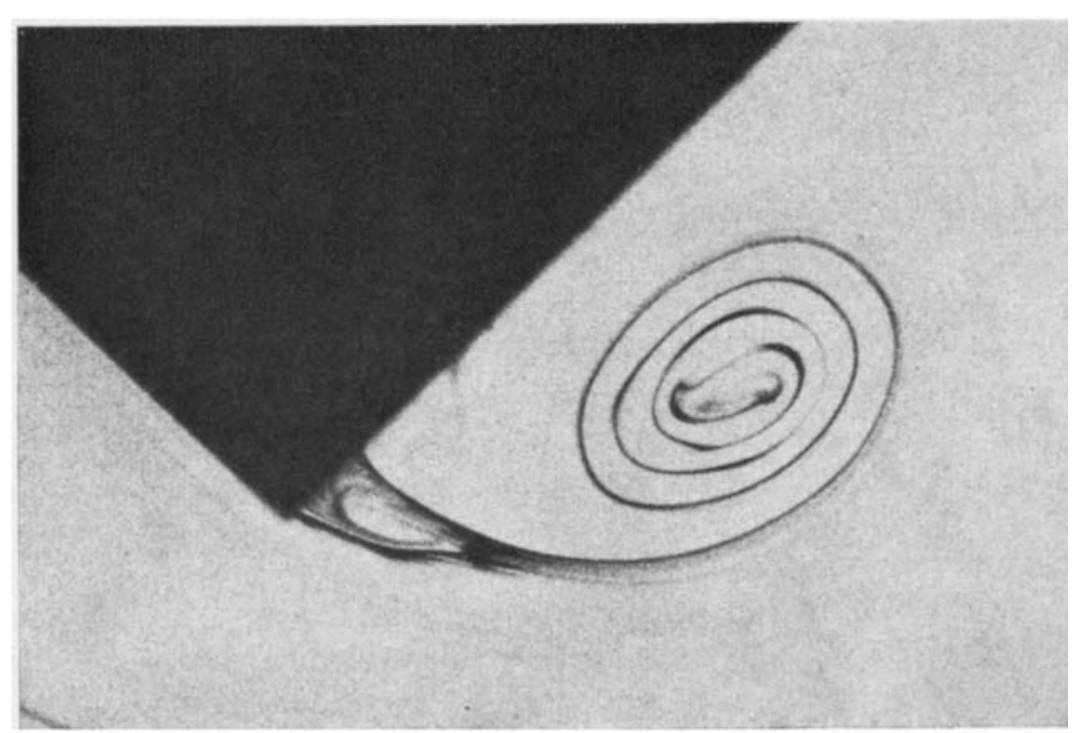

(b)

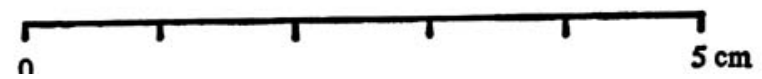

FigURE 10. (a) Enlargement of photo in figure $5(c): \beta=0.333\left(60^{\circ}\right), m=0, R e_{c}=1560$, $t=5 \mathrm{~s}, \boldsymbol{R e}_{\mathrm{s}}=967$. (b) Enlargement of photo in figure $9(e) ; \beta=\frac{1}{2}\left(90^{\circ}\right), m=0.24, R e_{\mathrm{c}}=5109$, $t=4.0 \mathrm{~s}, R e_{s}=3385$. 


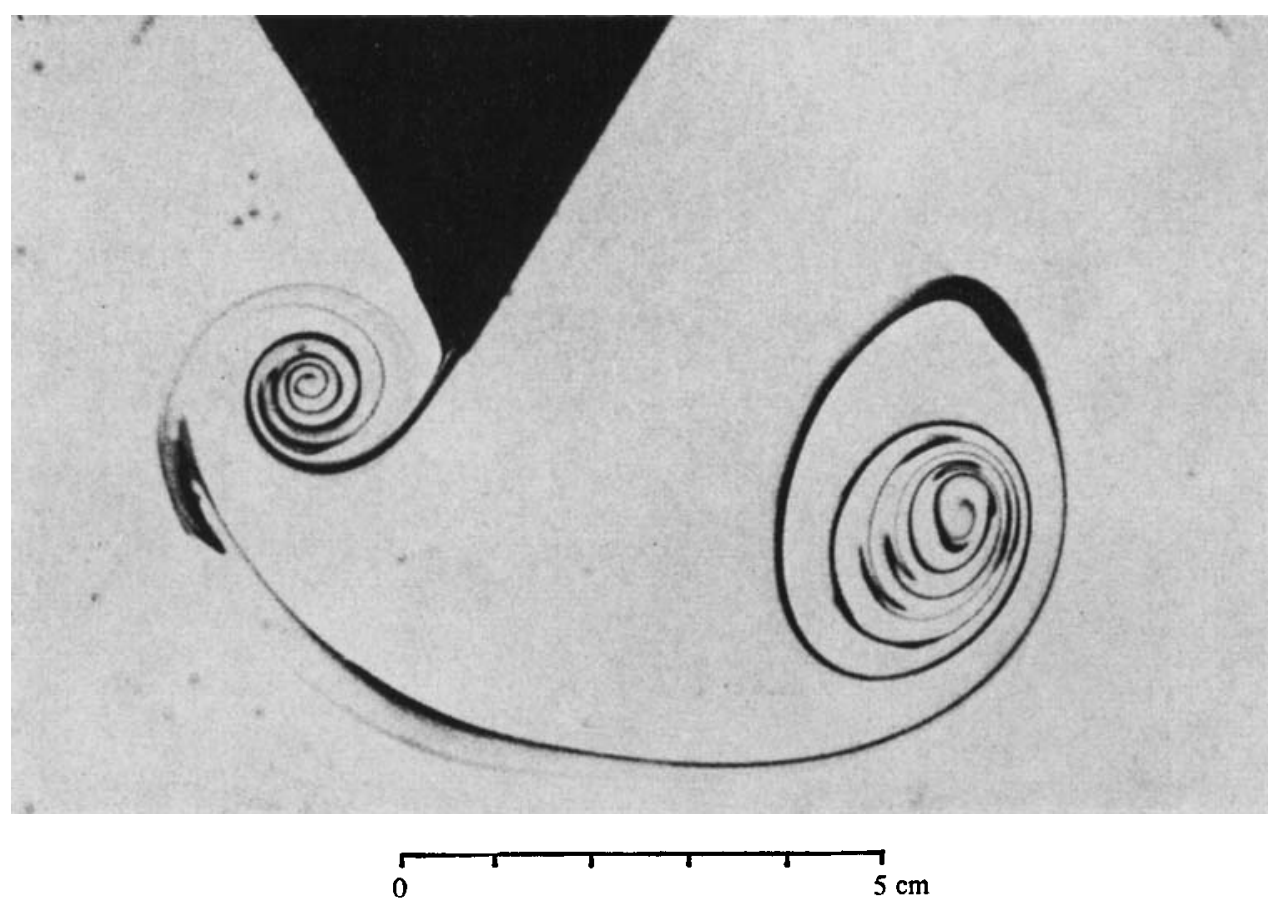

Frgure 11. Photo of dye in water showing vortex pair formed near wedge in starting-stopping flow. The generating flow is that for figure 6 with $\beta=0.333\left(60^{\circ}\right), m=0.45, R e_{c}=6621$. The flow was stopped at $t=5.96 \mathrm{~s}$ and this photo corresponds to $t=7.285 \mathrm{~s}$. 